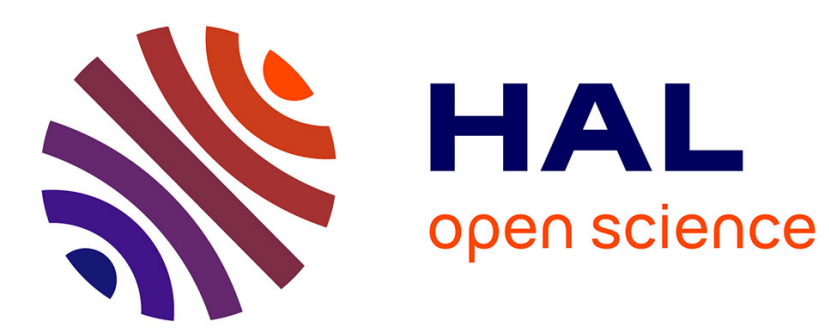

\title{
Modified Navier-Stokes equations for the outflow boundary conditions in hemodynamics
}

Grégory Arbia, Irene Vignon-Clementel, Tainyen Y. Hsia, Jean-Frédéric

Gerbeau

\section{- To cite this version:}

Grégory Arbia, Irene Vignon-Clementel, Tainyen Y. Hsia, Jean-Frédéric Gerbeau. Modified NavierStokes equations for the outflow boundary conditions in hemodynamics. European Journal of Mechanics - B/Fluids, 2016, 60, pp.175-188. 10.1016/j.euromechflu.2016.06.001 . hal-01328501

\section{HAL Id: hal-01328501 https://hal.science/hal-01328501}

Submitted on 8 Jun 2016

HAL is a multi-disciplinary open access archive for the deposit and dissemination of scientific research documents, whether they are published or not. The documents may come from teaching and research institutions in France or abroad, or from public or private research centers.
L'archive ouverte pluridisciplinaire HAL, est destinée au dépôt et à la diffusion de documents scientifiques de niveau recherche, publiés ou non, émanant des établissements d'enseignement et de recherche français ou étrangers, des laboratoires publics ou privés. 


\section{Modified Navier-Stokes equations for the outflow boundary conditions in hemodynamics*}

G. Arbia ${ }^{\mathrm{a}, \mathrm{b}, *}$, I.E. Vignon-Clementel ${ }^{\mathrm{a}, \mathrm{b}, *}$, T.-Y. Hsia ${ }^{\mathrm{c}}$, J-F. Gerbeau ${ }^{\mathrm{a}, \mathrm{b}}$, for the Modeling of Congenital Hearts Alliance (MOCHA) Investigators

${ }^{a}$ INRIA, Paris, France

${ }^{b}$ Sorbonne Universités, UPMC Univ Paris 6, Laboratoire Jacques-Louis Lions, Paris, France ${ }^{c}$ Centre for Cardiovascular Imaging, UCL Institute of Cardiovascular Science, and Great Ormond 8 Street Hospital for Children, NHS Foundation Trust, London, UK instabilities

${ }^{*}$ Authors contributed equally

${ }^{*}$ Accepted for publication in European Journal of Mechanics / B Fluids 
Abstract

We present a new approach for the outflow boundary conditions of Navier-

Stokes equations in hemodynamics. We first describe some existing 3D-0D cou-

pling methods and highlight benefits and disadvantages of each of them. We then introduce a new method that consists in adding a 3D artificial part where the Navier-Stokes equations are modified to obtain an equivalent energy balance to a standard coupling with a 3-element Windkessel model. We investigate theoretically the stability of the system and compare it to previously introduced methods. Finally we compare these coupling methods for numerical simulations of blood flow in three patient-specific models, which represent different flow regimes in the pulmonary and systemic circulations. The new method, especially in its hybrid form, is a possible alternative to existing methods. It can be in particular convenient in codes that do not allow users to implement non-standard boundary conditions.

\section{Introduction}

The three-dimensional Navier-Stokes simulation of blood flow in large vessels requires inlet and outlet boundary conditions that represent hemodynamics at these locations. However, in patient-specific settings, pressure or velocity are rarely clinically measured exactly there, or they can be part of the desired output [43]. Thus, boundary conditions are usually substituted for transmission conditions with reduced models of the rest of the circulation. This typically involves coupling to 1D models (e.g. [8, 15, 33, 41]), or simple lumped parameter 0D models (e.g. [18, $37,42,44])$ or closed loop lumped parameter 0D models (e.g. [3, 10, 25, 28, 30]). This coupling methodology transfers in various forms pressure and flow rate between 3D and reduced models, for which there is a loss of information. Moreover, reverse flow may occur in parts of or on entire coupling boundaries. This calls for 
careful coupling conditions, so that the scheme is stable, without altering too much the local flow dynamics.

A first coupling method consists in enforcing a uniform pressure obtained from the reduced model at the coupling boundary. When a variational formulation is used, e.g. with the finite element method, it is more natural to replace the pressure with the normal component of the normal stress. However stability analyses show that the convective term on the boundary can be responsible for numerical instabilities in the presence of reverse flow. Some Navier-Stokes formulations involve the total pressure in their natural boundary conditions (e.g. [5]). This has been shown to lead to an energetically stable coupling between 3D and reduced models of blood flow (e.g. $[16,17])$. Several authors have been expecting instabilities when the total pressure is not included in the transmission conditions but have not seen them numerically $[9,17]$. In formulations based on static pressure, a dissipative stabilization has been proposed to counteract the destabilizing effect of the convective boundary term (see [4]). We also refer to $[11,12]$ where similar ideas were introduced. A boundary condition based on enforcing the continuity of pressure on the one hand and of a linear combination of flow and energy fluxes on the other hand, has been proven to be energetically stable, but it does not necessarily conserve mass [14]. More recently, a local regularization of the fluid velocity along the tangential directions has been developed in [6]. In all these methods, the reduced model pressure is imposed as a uniform boundary condition for the Navier-Stokes equations. But in complex flow, such as with reverse flow, there is a priori no reason that the normal traction or the total pressure is uniform on a coupling boundary. For stabilized methods, the added term introduces some non-uniformity that has also no obvious reason to correspond to the physiological flow at hand. 
In this article, we propose a new method to handle the outflow boundary conditions, by coupling the 3D Navier-Stokes equations with another 3D compartment. This artificial compartment involves modified Navier-Stokes equations that mimic a three-element Windkessel model [19] (as an example of reduced model). The advantages of such an approach are that the coupling 1) does not enforce a uniform traction at the interface, 2) is energetically close to the usual 3D Navier-Stokes - Windkessel solution, without the potentially destabilizing convective boundary term, 3 ) is provably stable in the energy norm without needing a total pressure formulation. Besides, a strategy of adding an artificial 3D part can be very useful in a commercial code that does not allow the users to implement any 0D boundary conditions.

Our contributions with respect to the existing works considering a 3D compartment (e.g. [27]) are the following. First, our artificial compartment is more complex since it includes two different kinds of dissipation and one term representing an elastic potential energy. This allows us to mimic an RCR Windkessel model, which is important in our applications. Second, we investigate how this artificial compartment affects outflow instabilities typically encountered in hemodynamics; third, we make a numerical comparison of different outflow boundary conditions in realistic hemodynamics test cases.

In the next part of this article we present existing coupling methods, derive the corresponding energy balance. In this context, we present our new modified NavierStokes system and analyze its stability. The last part of the methods section is devoted to the numerical implementation. The results are then illustrated on different reverse flow situations, namely three patient-specific cases of numerical blood flow simulations in arteries in the pulmonary and the systemic sides for healthy or diseased configurations. The different methods are compared qualitatively, looking 
at the velocity fields at the coupling interface, and quantitatively on the flow and pressure. In some cases, the results are compared to a reference solution obtained on a larger geometry. Finally, a discussion with results of the literature is proposed and possible extensions are presented.

\section{Methods}

\subsection{Problem formulations}

Blood is assumed to be a Newtonian fluid flowing in a rigid domain $\Omega_{1}$. The incompressible Navier-Stokes equations are solved with no-slip condition on the wall $\Gamma$ and with a Dirichlet boundary condition on the velocity at the inlet $\Gamma_{\text {in }}$ (the notation is defined in Figure 1):

$$
\begin{aligned}
\rho \frac{\partial \boldsymbol{u}}{\partial t}+\rho(\boldsymbol{u} \cdot \nabla \boldsymbol{u})+\nabla p-2 \mu \nabla \cdot \boldsymbol{\varepsilon}(\boldsymbol{u}) & =0 \\
\nabla \cdot \boldsymbol{u} & =0 \\
\boldsymbol{u}_{\left.\right|_{\Gamma}} & =0 \\
\boldsymbol{u}_{\left.\right|_{\text {in }}} & =\boldsymbol{u}_{\text {in }}
\end{aligned}
$$

where $\boldsymbol{u}: \Omega_{1} \times \mathbb{R}^{+} \rightarrow \mathbb{R}^{3}$ is the velocity, $p: \Omega_{1} \times \mathbb{R}^{+} \rightarrow \mathbb{R}$ is the pressure, and $\boldsymbol{\varepsilon}(\boldsymbol{u})=\frac{1}{2}\left(\nabla \boldsymbol{u}+\nabla \boldsymbol{u}^{T}\right)$ denotes the strain rate tensor.

The system (1)-(4) has to be complemented with boundary conditions at the outlet $\Gamma_{\text {out }}$, where the Navier-Stokes equations are coupled to a reduced-order model which takes into account the rest of the vessels. Here the names "inlet" and "outlet" are chosen for convenience, but any of these can have some positive or negative flow rate over time. Three possible approaches that have been proposed in the literature are presented below. Then, the main contribution of this study, based on a $3 \mathrm{D}-3 \mathrm{D}$ coupling, is introduced. 


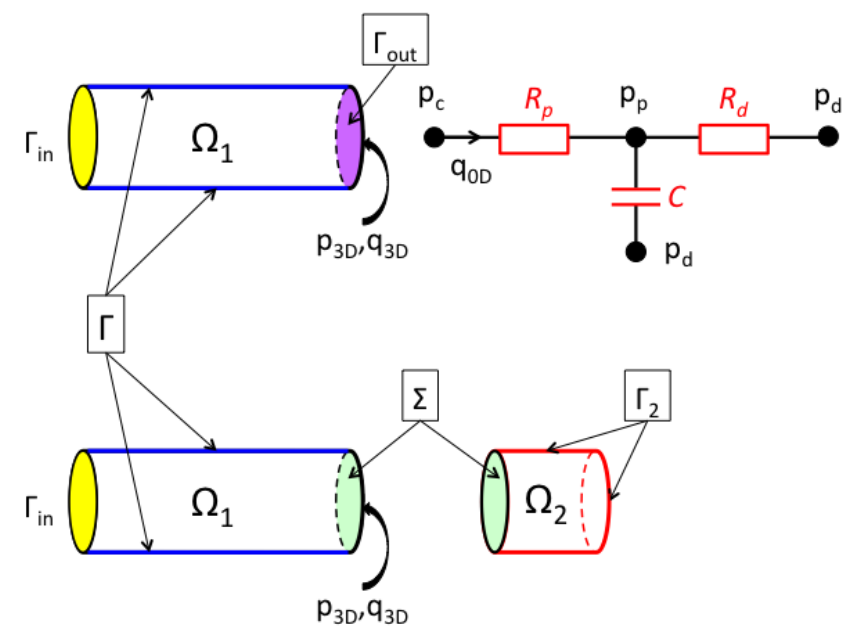

Figure 1: top: 3D-0D coupling scheme, bottom: 3D-3D coupling scheme.

\subsection{Review of three existing approaches}

Formulation 1: 3D-OD . The most common idea consists in enforcing a uniform normal stress at the outlet, equal to a pressure $p_{\mathrm{c}}$ given by a reduced model:

$$
\boldsymbol{\sigma} \cdot \boldsymbol{n}=-p_{\mathrm{c}} \boldsymbol{n}, \quad \text { on } \Gamma_{\text {out }},
$$

where $\sigma=-p \mathbf{I}+2 \mu \varepsilon(\boldsymbol{u})$. In a finite element framework, this is easily done with a Neumann boundary condition. The advantage of this formulation is its simplicity. Although stable in many practical situations, instabilities can be observed in presence of complex reverse flow. It will be shown in Section 2.4 that an uncontrolled term appears in the energy balance. This is a possible reason of the observed instabilities. In addition, enforcing a uniform normal stress on a section where the flow is complex may generate spurious vortices that can also lead to instabilities. 
Formulation 2: 3D-0D-Stab . To cure the instabilities that may appear during backflow with Formulation 1, a stabilization method was proposed in [4]. This approach consists in modifying (5) as follows (here written in a slightly more general form):

$$
\boldsymbol{\sigma} \cdot \boldsymbol{n}=-p_{c} \boldsymbol{n}-\rho \theta(\boldsymbol{u} \cdot \boldsymbol{n})_{-} \boldsymbol{u}, \quad \text { on } \Gamma_{\text {out }},
$$

where $(\boldsymbol{u} \cdot \boldsymbol{n})_{-}$is equal to $-(\boldsymbol{u} \cdot \boldsymbol{n})$ if $\boldsymbol{u} \cdot \boldsymbol{n} \leq 0$, and is equal to 0 if not, and where $\theta$ has to be fixed.

This method is simple to implement and can be shown to be stable in the energy norm for $\theta \geq 0.5$. In practice, it proves to be very efficient at reducing the outlet instabilities. It was successfully tested in particular in $[29,36]$. Two situations have to be distinguished here. When the backflow is due to spurious vortices induced by the presence of an artificial boundary, this effect is desirable. But it is well-known that a physical backflow can appear in some vessels during diastole. In those cases, the stabilization term artificially takes out of the system an energy that should enter through the outlet.

Formulation 3: 3D-0D-Ptot . Another approach considers a formulation of the Navier-Stokes equations that involves the total pressure (e.g. [5, 22]):

$$
\boldsymbol{\sigma} \cdot \boldsymbol{n}-\frac{\rho}{2}|\boldsymbol{u}|^{2} \boldsymbol{n}=-p_{\mathrm{c}} \boldsymbol{n}, \quad \text { on } \Gamma_{\text {out }}
$$

This formulation, advocated in particular in $[16,17]$, can be proved to be stable in the energy norm (see below). But in blood flow, the total stress is far from being uniform on a section. Note that this is even not true for Poiseuille or Womersley flows. As a consequence, this formulation leads to large spurious velocity vectors, even for simple configurations [22]. As in Formulation 1, spurious vortices can be generated at this interface and can eventually trigger instabilities. 


\subsection{A new formulation}

Formulation $4 a: 3 D-3 D$. We propose a possible alternative to the previous approaches. System (1)-(4) is coupled to a modified Navier-Stokes system. Instead of a 0D model, an artificial 3D domain $\Omega_{2}$ is added to the 3D domain of interest $\Omega_{1}$ (bottom of Figure 1). In $\Omega_{2}$, the Navier-Stokes equations are modified by adding terms to recover an energy balance similar to the one obtained with Formulation 3. The resulting system can be written in a compact form in $\Omega_{1} \cup \Omega_{2}$ :

$$
\begin{aligned}
\rho \frac{\partial \boldsymbol{u}}{\partial t}+\rho \boldsymbol{u} \cdot \nabla \boldsymbol{u}+\nabla p-2 \mu \nabla \cdot \boldsymbol{\varepsilon}(\boldsymbol{u})+\gamma \boldsymbol{u}+\rho \frac{\boldsymbol{u}}{2} \nabla \cdot \boldsymbol{u} & =0 \\
\alpha \frac{\partial p}{\partial t}+\beta p+\nabla \cdot \boldsymbol{u} & =0 \\
\boldsymbol{u}_{\mid Г \curlyvee\left\ulcorner ~_{2}\right.} & =0
\end{aligned}
$$

where $\alpha, \beta$ and $\gamma$ vanish in $\Omega_{1}$, to recover the standard equations (1)-(4), and are positive in $\Omega_{2}$. As indicated in Figure 1, the surface $\Gamma_{2}$ consists of the wall of the added artificial volume, i.e. $\Gamma_{2}=\partial \Omega_{2} \backslash \Sigma$. In other words the added artificial surface acts as a "cork".

Parameter $\alpha$ is a distributed version of the capacitance $(C)$ in the Windkessel model. Parameters $\gamma$ and $1 / \beta$ play the role of the proximal $\left(R_{p}\right)$ and distal $\left(R_{d}\right)$ resistances respectively. Their values will be discussed in Section 2.4. Note that the term $\beta p$ acts as a sink, whereas the term $\alpha \partial_{t} p$ acts as a sink when the pressure increases and as a source when the pressure decreases. The additional term $\rho \frac{\boldsymbol{u}}{2} \nabla \cdot \boldsymbol{u}$ is necessary to ensure stability in the energy norm, because the fluid is no longer incompressible in the artificial domain $\Omega_{2}$. The equations in the two domains $\Omega_{1}$ and $\Omega_{2}$ are coupled through the usual transmission conditions, with $\boldsymbol{n}$ being defined on $\Sigma$ as going from $\Omega_{1}$ to $\Omega_{2}$ :

$$
\boldsymbol{u}_{1}=\boldsymbol{u}_{2}, \text { and } \boldsymbol{\sigma}_{1} \cdot \boldsymbol{n}=\boldsymbol{\sigma}_{2} \cdot \boldsymbol{n}, \quad \text { on } \Sigma,
$$


which are automatically satisfied when a standard variational formulation of equations (8)-(10) is set on the whole domain $\Omega_{1} \cup \Omega_{2}$.

Contrary to what happened with the three previous formulations, the information coming from the "downstream part" is not uniform on the outflow section $\Gamma_{\text {out }}$. Contrary to Formulation 1, an inequality can be proved to control the energy of the system. Compared to Formulation 2, it can let energy enter into the system through $\Gamma_{\text {out }}$ in the presence of a physical backflow.

Formulation $4 b$. As a variant, we also propose a hybrid 3D-3D-0D coupling method where the Navier-Stokes equations are coupled to the modified Navier-Stokes model, which is itself coupled to a 3-element Windkessel reduced model, as shown in Figure 2 .

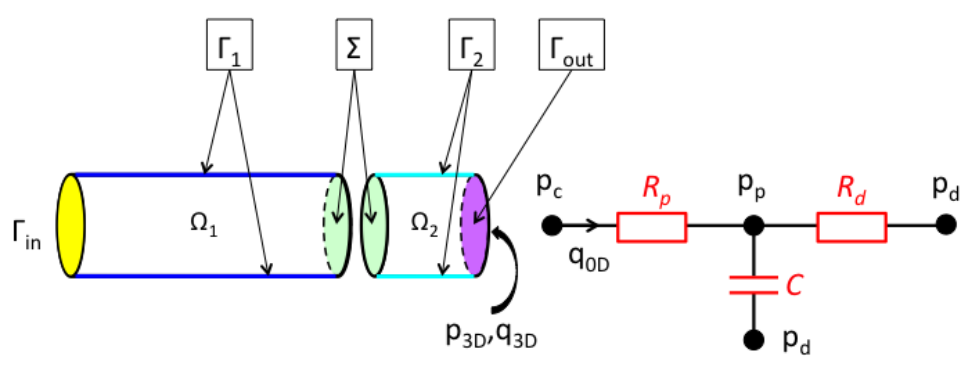

Figure 2: 3D-3D-0D coupling approach (formulation $4 \mathrm{~b}$ ), where $\Omega_{1}$ is the domain of interest, $\Omega_{2}$ is an artificial added part, coupled to a 3-element Windkessel model. 
In the whole domain $\Omega_{1} \cup \Omega_{2}$,

$$
\begin{aligned}
\rho \frac{\partial \boldsymbol{u}}{\partial t}+\rho(\boldsymbol{u} \cdot \nabla \boldsymbol{u})+\nabla p-2 \mu \nabla \cdot \boldsymbol{\varepsilon}(\boldsymbol{u})+\gamma \boldsymbol{u} & =0 \\
\nabla \cdot \boldsymbol{u} & =0 \\
\boldsymbol{u}_{\left.\right|_{\Gamma_{1} \cup \Gamma_{2}}} & =0 \\
\boldsymbol{u}_{\left.\right|_{\Gamma_{\mathrm{in}}}} & =\boldsymbol{u}_{\text {in }}
\end{aligned}
$$

174 where $\gamma=0$ in $\Omega_{1}$, with the usual transmission conditions (11). We finally couple 175 a 3-element Windkessel model to the system (12)-(15) with the 3D-0D coupling 176 method,

$$
\boldsymbol{\sigma} \cdot \boldsymbol{n}=-p_{\mathrm{c}} \boldsymbol{n}, \quad \text { on } \Gamma_{\text {out }},
$$

177 2.4. Stability analysis

178 In this section, the energy equations of the various formulations presented above 179 are derived. Without loss of generality, the reduced-order model providing the pressure $p_{c}$ in Formulations 1, 2 and 3, is assumed for now to result from a standard

181 RCR Windkessel model (Figure 1, top):

$$
\begin{aligned}
C \frac{d p_{p}}{d t}+\frac{p_{p}-p_{d}}{R_{d}} & =q \\
p_{c}-p_{p} & =R_{p} q
\end{aligned}
$$

182 where $q=\int_{\Gamma_{\text {out }}} \boldsymbol{u} \cdot \boldsymbol{n} d s$. For the sake of simplicity, we assume that the distal pressure ${ }_{183} p_{d}$ is constant and equal to zero.

184

185 Multiplying (18) by $q$ and (17) by $p_{p}$ leads to the energy equation of the lumped 186 parameter model:

$$
p_{c} q=C \frac{d}{d t}\left(\frac{p_{p}^{2}}{2}\right)+R_{p} q^{2}+\frac{p_{p}^{2}}{R_{d}}
$$


We define the kinetic energy $E_{K_{\Omega_{1}}}$, the viscous power $P_{V_{\Omega_{1}}}$ and the energy $P_{\text {in }}$ entering the domain through $\Gamma_{\text {in }}$, respectively by:

$$
\begin{array}{r}
E_{K_{\Omega_{1}}}=\int_{\Omega_{1}} \frac{\rho}{2}|\boldsymbol{u}|^{2} d x, \\
P_{V_{\Omega_{1}}}=2 \mu \int_{\Omega_{1}} \varepsilon(\boldsymbol{u}): \varepsilon(\boldsymbol{u}) d x, \\
P_{\text {in }}=\int_{\Gamma_{\text {in }}} \boldsymbol{\sigma} \cdot \boldsymbol{n} \cdot \boldsymbol{u} d s-\rho \int_{\Gamma_{\text {in }}} \frac{|\boldsymbol{u}|^{2}}{2} \boldsymbol{n} \cdot \boldsymbol{u} d s .
\end{array}
$$

$$
\frac{d}{d t} E_{K_{\Omega_{1}}}+P_{V_{\Omega_{1}}}+C \frac{d}{d t} \frac{p_{p}^{2}}{2}+\frac{p_{p}^{2}}{R_{d}}+R_{p} q^{2}=P_{\text {in }}-\rho \int_{\Gamma_{\text {out }}} \frac{|\boldsymbol{u}|^{2}}{2} \boldsymbol{n} \cdot \boldsymbol{u} d s
$$

$$
\frac{d}{d t} \int_{\Omega_{1}} \frac{\rho}{2}|\boldsymbol{u}|^{2} d x+\rho \int_{\Omega_{1}} \boldsymbol{u} \cdot \nabla\left(\frac{|\boldsymbol{u}|^{2}}{2}\right) d x+2 \mu \int_{\Omega_{1}} \boldsymbol{\varepsilon}(\boldsymbol{u}): \boldsymbol{\varepsilon}(\boldsymbol{u}) d x+\int_{\Omega_{1}} \nabla p \cdot \boldsymbol{u} d x=0
$$

B9 Using (2) and the no-slip condition on the wall, the energy equation in $\Omega_{1}$ reads:

$$
\frac{d}{d t} E_{K_{\Omega_{1}}}+P_{V_{\Omega_{1}}}=P_{\mathrm{in}}+\int_{\Gamma_{\text {out }}} \boldsymbol{\sigma} \cdot \boldsymbol{n} \cdot \boldsymbol{u} d s-\rho \int_{\Gamma_{\text {out }}} \frac{|\boldsymbol{u}|^{2}}{2} \boldsymbol{n} \cdot \boldsymbol{u} d s
$$

Considering the coupling condition (5) and the energy equation of the OD model (19), the global energy balance of the 3D-0D coupling method is obtained:

In the presence of reverse flow at an outlet $(\boldsymbol{u} \cdot \boldsymbol{n}<0)$ the last term may thus have a destabilizing effect.

Energy balance of Formulation 2 (3D-0D-Stab coupling). With the same computation as in Formulation 1, but considering (6) instead of (5) as a coupling condition, the energy equation of Formulation 2 reads:

$\frac{d}{d t} E_{K_{\Omega_{1}}}+P_{V_{\Omega_{1}}}+C \frac{d}{d t} \frac{p_{p}^{2}}{2}+\frac{p_{p}^{2}}{R_{d}}+R_{p} q^{2}=\left\{\begin{array}{l}P_{\text {in }}-\frac{\rho}{2} \int_{\Gamma_{\text {out }}}|\boldsymbol{u}|^{2}(\boldsymbol{u} \cdot \boldsymbol{n}) d s, \text { if } \boldsymbol{u} \cdot \boldsymbol{n} \geq 0 \\ P_{\text {in }}-\rho\left(\frac{1}{2}-\theta\right) \int_{\Gamma_{\text {out }}}|\boldsymbol{u}|^{2}(\boldsymbol{u} \cdot \boldsymbol{n}) d s, \text { if } \boldsymbol{u} \cdot \boldsymbol{n}<0\end{array}\right.$ 
This formulation is therefore stable in the energy norm for $\theta \geq 0.5$. If $\theta=0.5$, as in $[29,36]$, the potentially destabilizing term of (24) is exactly balanced by the artificial dissipation when $\boldsymbol{u} \cdot \boldsymbol{n}<0$. In some publications, a stronger dissipation is chosen (e.g. $\theta=1$ in [4]).

Energy balance of Formulation 3 (3D-0D-Ptot coupling): . When coupling is done with the total pressure (7), the potentially destabilizing term of (24) disappears in the energy balance:

$$
\frac{d}{d t} E_{K_{\Omega_{1}}}+P_{V_{\Omega_{1}}}+C \frac{d}{d t} \frac{p_{p}^{2}}{2}+\frac{p_{p}^{2}}{R_{d}}+R_{p} \boldsymbol{q}^{2}=P_{\mathrm{in}}
$$

This formulation is therefore stable in the energy norm.

Energy balance of Formulation 4 a (3D-3D coupling): As in Formulation 1, the energy equation in the domain of interest $\Omega_{1}$ reads:

$$
\frac{d}{d t} E_{K_{\Omega_{1}}}+P_{V_{\Omega_{1}}}=P_{\text {in }}^{\Omega_{1}}+\int_{\Sigma}\left(\boldsymbol{\sigma} \cdot \boldsymbol{n} \cdot \boldsymbol{u}-\rho \frac{|\boldsymbol{u}|^{2}}{2} \boldsymbol{n} \cdot \boldsymbol{u}\right) d s
$$

Similarly, in the artificial domain $\Omega_{2}$, from the modified Navier-Stokes equation (8)-(9)-(10):

$$
\frac{d}{d t} E_{K_{\Omega_{2}}}+P_{V_{\Omega_{2}}}+\gamma \int_{\Omega_{2}}|\boldsymbol{u}|^{2} d x-\int_{\Omega_{2}} p \nabla \cdot \boldsymbol{u} d x=-\int_{\Sigma} \boldsymbol{\sigma} \cdot \boldsymbol{n} \cdot \boldsymbol{u} d s+\rho \int_{\Sigma} \frac{|\boldsymbol{u}|^{2}}{2} \boldsymbol{n} \cdot \boldsymbol{u} d s
$$

Using equation (9),

$$
\begin{aligned}
& \frac{d}{d t} E_{K_{\Omega_{2}}}+P_{V_{\Omega_{2}}}+\alpha \frac{d}{d t} \int_{\Omega_{2}} \frac{p^{2}}{2} d x+\beta \int_{\Omega_{2}} p^{2} d x+\gamma \int_{\Omega_{2}}|\boldsymbol{u}|^{2} d x \\
& =-\int_{\Sigma} \boldsymbol{\sigma} \cdot \boldsymbol{n} \cdot \boldsymbol{u} d s+\rho \int_{\Sigma} \frac{|\boldsymbol{u}|^{2}}{2} \boldsymbol{n} \cdot \boldsymbol{u} d s
\end{aligned}
$$

Adding these two relations, the boundary terms in the right-hand side cancel out, due to the transmission condition (11), and the energy balance of Formulation 4a is obtained:

$$
\frac{d}{d t} E_{K}^{\Omega_{1} \cup \Omega_{2}}+P_{V}^{\Omega_{1} \cup \Omega_{2}}+\alpha \frac{d}{d t} \int_{\Omega_{2}} \frac{p^{2}}{2} d x+\beta \int_{\Omega_{2}} p^{2} d x+\gamma \int_{\Omega_{2}}|\boldsymbol{u}|^{2} d x=P_{\mathrm{in}}^{\Omega_{1}} .
$$



by:

$$
p_{c}^{n+1}=\frac{\frac{\tau}{\Delta t} p_{p}^{n}+R_{d} q^{n}}{1+\frac{\tau}{\Delta t}}+R_{p} q^{n} .
$$

The 3D formulations are discretized with a first-order time scheme and P1/P1 stabilized finite element [35]. The nonlinear advection term of the Navier-Stokes 


$$
\begin{array}{r}
A_{2}^{\Omega}\left(\boldsymbol{u}_{h}^{n} ;\left(\boldsymbol{u}_{h}^{n+1}, p_{h}^{n+1}\right),\left(\boldsymbol{v}_{h}, q_{h}\right)\right)=\int_{\Omega}\left(\frac{\rho}{\Delta t} \boldsymbol{u}_{h}^{n+1}+\rho\left(\nabla \times \boldsymbol{u}_{h}^{n+1}\right) \times \boldsymbol{u}_{h}^{n}\right) \cdot \boldsymbol{v}_{h} d x \\
-\int_{\Omega} \frac{\rho}{2} \boldsymbol{u}_{h}^{n+1} \cdot \boldsymbol{u}_{h}^{n} \nabla \cdot \boldsymbol{v}_{h} d x+\int_{\Omega_{1}} \mu \nabla \boldsymbol{u}_{h}^{n+1}: \nabla \boldsymbol{v}_{h} d x-\int_{\Omega}\left(p_{h}^{n+1} \nabla \cdot \boldsymbol{v}_{h}+q_{h} \nabla \cdot \boldsymbol{u}_{h}^{n+1}\right) d x .(38)
\end{array}
$$

$$
\begin{array}{r}
A_{1}^{\Omega}\left(\boldsymbol{u}_{h}^{n} ;\left(\boldsymbol{u}_{h}^{n+1}, p_{h}^{n+1}\right),\left(\boldsymbol{v}_{h}, q_{h}\right)\right)=\int_{\Omega}\left(\frac{\rho}{\Delta t} \boldsymbol{u}_{h}^{n+1}+\rho \boldsymbol{u}_{h}^{n} \cdot \nabla \boldsymbol{u}_{h}^{n+1}\right) \cdot \boldsymbol{v}_{h} d x \\
+\int_{\Omega} \mu \nabla \boldsymbol{u}_{h}^{n+1}: \nabla \boldsymbol{v}_{h} d x-\int_{\Omega}\left(p_{h}^{n+1} \nabla \cdot \boldsymbol{v}_{h}+q_{h} \nabla \cdot \boldsymbol{u}_{h}^{n+1}\right) d x,
\end{array}
$$

the variational formulation of the 3D-0D method reads: Find $\boldsymbol{u}_{h}^{n+1}$ and $p_{h}^{n+1}$ such hat for every $\boldsymbol{v}_{h}$ and $q_{h}$,

$$
A_{1}^{\Omega_{1}}\left(\boldsymbol{u}_{h}^{n} ;\left(\boldsymbol{u}_{h}^{n+1}, p_{h}^{n+1}\right),\left(\boldsymbol{v}_{h}, q_{h}\right)\right)=\int_{\Omega_{1}} \frac{\rho}{\Delta t} \boldsymbol{u}_{h}^{n} \cdot \boldsymbol{v}_{h} d x-\int_{\Gamma_{\text {out }}} p_{c}^{n+1} \boldsymbol{n} \cdot \boldsymbol{v}_{h} d s
$$

Remark 2.1. Note that the weak form of the viscous term is based on the relation $\mu \Delta \boldsymbol{u}=2 \mu \nabla \cdot \boldsymbol{\varepsilon}(\boldsymbol{u})$, valid for an incompressible fluid. The natural condition corresponding to this formulation is compatible with Poiseuille and Womersley flows contrary to the one based on $\varepsilon(\boldsymbol{u})($ e.g. [22]). We made this choice to avoid perturbations of the velocity field at the outlet for these basic flows, but the formulation based on $\varepsilon(\boldsymbol{u})$ can also be used in practice.

Formulation 2 (3D-0D-Stab ). Considering the bilinear form (35) and boundary conditions (6) with $\theta=0.5$, the 3D-0D-Stab method variational formulation reads:

Find $\boldsymbol{u}_{h}^{n+1}$ and $p_{h}^{n+1}$ such that for every $\boldsymbol{v}_{h}$ and $q_{h}$,

$$
\begin{gathered}
A_{1}^{\Omega_{1}}\left(\boldsymbol{u}_{h}^{n} ;\left(\boldsymbol{u}_{h}^{n+1}, p_{h}^{n+1}\right),\left(\boldsymbol{v}_{h}, q_{h}\right)\right)= \\
\int_{\Omega_{1}} \frac{\rho}{\Delta t} \boldsymbol{u}_{h}^{n} \cdot \boldsymbol{v}_{h} d x-\int_{\Gamma_{\text {out }}} p_{c}^{n+1} \boldsymbol{n} \cdot \boldsymbol{v}_{h} d s-\int_{\Gamma_{\text {out }}} \frac{\rho}{2}\left(\boldsymbol{u}_{h}^{n} \cdot \boldsymbol{n}\right)_{-}\left(\boldsymbol{u}_{h}^{n+1} \cdot \boldsymbol{v}_{h}\right) d s
\end{gathered}
$$


The variational formulation of the 3D-0D-Ptot method reads: Find $\boldsymbol{u}_{h}^{n+1}$ and $p_{h}^{n+1}$ such that for every $\boldsymbol{v}_{h}$ and $q_{h}$,

$$
A_{2}^{\Omega_{1}}\left(\boldsymbol{u}_{h}^{n} ;\left(\boldsymbol{u}_{h}^{n+1}, p_{h}^{n+1}\right),\left(\boldsymbol{v}_{h}, q_{h}\right)\right)=\int_{\Omega_{1}} \frac{\rho}{\Delta t} \boldsymbol{u}_{h}^{n} \cdot \boldsymbol{v}_{h} d x-\int_{\Gamma_{\text {out }}} p_{c}^{n+1} \boldsymbol{n} \cdot \boldsymbol{v}_{h} d s
$$

Remark 2.2. In this formulation, the total pressure only appears in the boundary condition but the pressure unknown is still the static pressure. It is also possible to consider a formulation, based on the relation $\boldsymbol{u} \cdot \nabla \boldsymbol{u}^{T}=\nabla\left(\frac{|\boldsymbol{u}|^{2}}{2}\right)$, where the static pressure is replaced by the total pressure as an unknown [22].

Formulation $4 a(3 D-3 D)$. Defining the bilinear form:

$$
\begin{array}{r}
A_{3}^{\Omega}\left(\boldsymbol{u}_{h}^{n} ;\left(\boldsymbol{u}_{h}^{n+1}, p_{h}^{n+1}\right),\left(\boldsymbol{v}_{h}, q_{h}\right)\right)=\int_{\Omega}\left(\frac{\rho}{\Delta t} \boldsymbol{u}_{h}^{n+1}+\rho \boldsymbol{u}_{h}^{n} \cdot \nabla \boldsymbol{u}_{h}^{n+1}\right) \cdot \boldsymbol{v}_{h} d x \\
+\int_{\Omega} \mu \nabla \boldsymbol{u}_{h}^{n+1}: \nabla \boldsymbol{v}_{h} d x-\int_{\Omega}\left(p_{h}^{n+1} \nabla \cdot \boldsymbol{v}_{h}+q_{h} \nabla \cdot \boldsymbol{u}_{h}^{n+1}\right) d x \\
+\int_{\Omega} \gamma \boldsymbol{u}_{h}^{n+1} \cdot \boldsymbol{v}_{h} d x+\int_{\Omega} \frac{\rho}{2} \boldsymbol{u}_{h}^{n+1} \cdot \boldsymbol{v}_{h} \nabla \cdot \boldsymbol{u}_{h}^{n} d x-\int_{\Omega}\left(\frac{\alpha}{\Delta t}+\beta\right) p_{h}^{n+1} q_{h} d x
\end{array}
$$

where, $\alpha=\beta=\gamma=0$ in $\Omega_{1}$.

The variational formulation of the 3D-3D method reads: Find $\boldsymbol{u}_{h}^{n+1}$ and $p_{h}^{n+1}$ such that for every $\boldsymbol{v}_{h}$ and $q_{h}$,

$$
A_{3}^{\Omega_{1} \cup \Omega_{2}}\left(\boldsymbol{u}_{h}^{n} ;\left(\boldsymbol{u}_{h}^{n+1}, p_{h}^{n+1}\right),\left(\boldsymbol{v}_{h}, q_{h}\right)\right)=\int_{\Omega_{1} \cup \Omega_{2}}\left(\frac{\rho}{\Delta t} \boldsymbol{u}_{h}^{n} \cdot \boldsymbol{v}_{h}-\frac{\alpha}{\Delta t} p_{h}^{n} q_{h}\right) d x
$$

The variational formulation of Formulation $4 b$ (3D-3D-0D) is the same with the addition on the right hand side of $-\int_{\Gamma_{\text {out }}} p_{c}^{n+1} \boldsymbol{n} \cdot \boldsymbol{v}_{h} \mathrm{dx}$, and taking $\alpha=0, \beta=0$.

\section{Results}

In this section we present results of all these coupling methods for patient-specific cases in the systemic and the pulmonary sides where the flow regimes are very different. The first case is a typical case of flow in pulmonary arteries: the flow 
field is not so complex, and yet prescribed inlet back flow generates stability issues. For the second case the main advantages are first that the effect of artificially cutting the geometry can be assessed, and second that, due to the bifurcation close to the inlet, it is a case of more complex flow, although at the inlet no back flow is imposed. Finally, the third case tests a typical systemic hemodynamics scenario: instabilities there are generally observed during deceleration after peak systole. These three cases thus reflect the diversity of typical numerical instabilities seen in cardiovascular simulations. Through them, we highlight differences, benefits and disadvantages in the instability treatments.

For each case, the choice of Windkessel parameters is described. For the 3D-3D model, parameters were chosen following equations 31, given the specific added volume dimensions. Finally, parameters of the hybrid 3D-3D-0D method were defined as follows: in the artificial parts, $\gamma$ is chosen as $10 \%$ of the $\gamma$ in the 3D-3D model, while correspondingly the proximal resistance of the Windkessel model is decreased by $10 \%$ compared to the original value.

\subsection{Adult patient-specific pulmonary artery: a case with inlet backflow}

This patient specific geometrical model is an adult pulmonary artery (PA). Velocity is prescribed at the inlet of the model, as a plug profile following the flow tracing of Figure 3. There is around $16 \%$ of reverse flow. The maximum flow rate is around $378 \mathrm{~cm}^{3} / \mathrm{s}$, leading to a highest Reynolds number of 2400 . During the deceleration after systole, physical reverse flow occurs with a minimal flow rate around $-66 \mathrm{~cm}^{3} / \mathrm{s}$. Figure 3 also shows on the left-hand side the original geometrical model containing $107 \mathrm{~K}$ tetrahedra, with both outlets coupled to 3 -element Windkessel models by successively the 3D-0D, 3D-0D-Stab and 3D-0D-Ptot methods. 
On the right-hand side, the geometrical model with the added artificial parts consists of $127 \mathrm{~K}$ tetrahedra: there the coupling is done with the 3D-3D and 3D-3D-0D methods.

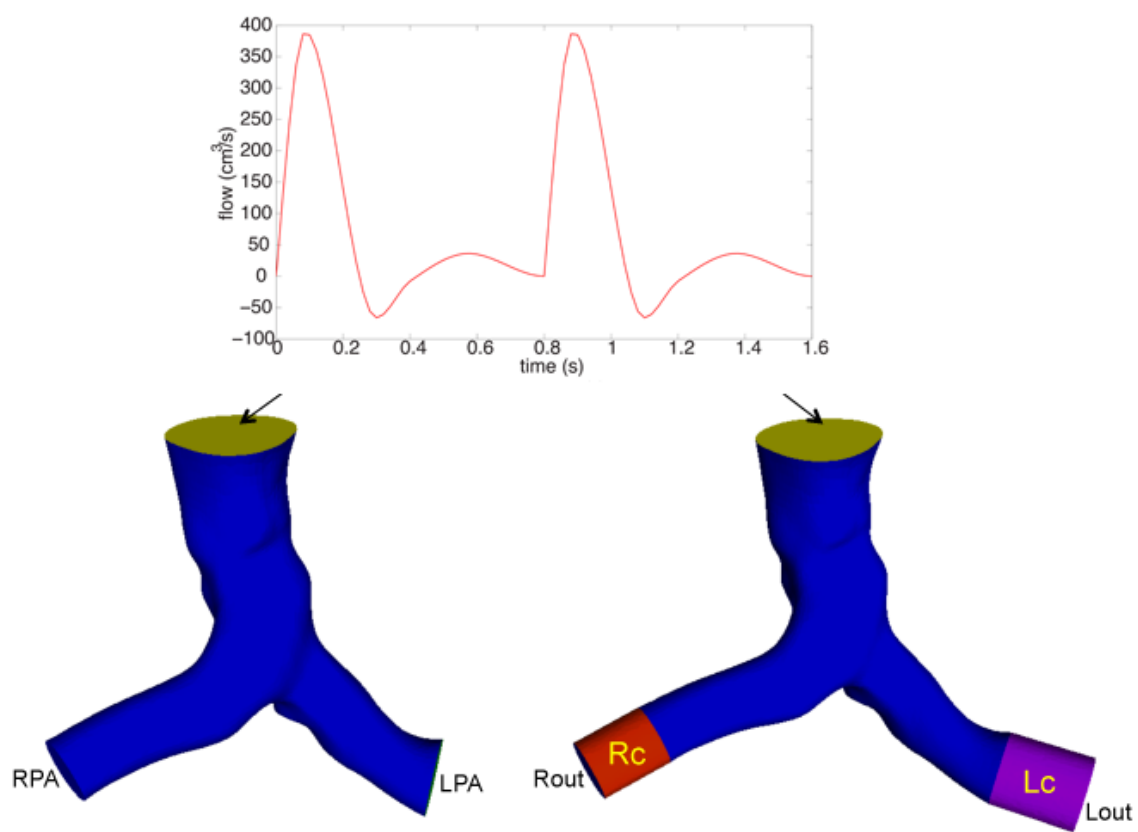

Figure 3: Original geometrical model in blue (left) and geometrical model with the added outlet 3D artificial parts in red and purple (right). Inlet flow tracing over two cardiac cycles.

Table 1 summarizes the parameters of the different coupling methods. On the lefthand side, the Windkessel model parameters are the ones of the 3D-0D, 3D-0DStab and 3D-0D-Ptot coupling approaches. They were chosen to represent physiological PA pressure pulses, assuming symmetry between the two lung vasculatures [21]. The 3D-3D (right) and 3D-3D-0D (below) parameters in the artificial added parts are displayed, given that the added volumes are such that $V, S$ and $L$ are respectively $5 \mathrm{~cm}^{3}, 2.5 \mathrm{~cm}^{2}$, and $2 \mathrm{~cm}$ on both sides. 


\begin{tabular}{|c|c|c||c|c|c|}
\hline & LPA & RPA & & Lc & Rc \\
\hline$R_{p}$ & $410^{1}$ & $410^{1}$ & $\gamma$ & $4.910^{1}$ & $4.910^{1}$ \\
\hline$C$ & $110^{-3}$ & $110^{-3}$ & $\alpha$ & $210^{-4}$ & $210^{-4}$ \\
\hline$R_{d}$ & $310^{2}$ & $310^{2}$ & $\beta$ & $6.710^{-4}$ & $6.710^{-4}$ \\
\hline
\end{tabular}

\begin{tabular}{|c|c|c||c|c|c|}
\hline & Lout & Rout & & Lc & Rc \\
\hline$R_{p}$ & $3.610^{1}$ & $3.610^{1}$ & $\gamma$ & 4.9 & 4.9 \\
\hline$C$ & $110^{-3}$ & $110^{-3}$ & $\alpha$ & 0 & 0 \\
\hline$R_{d}$ & $310^{2}$ & $310^{2}$ & $\beta$ & 0 & 0 \\
\hline
\end{tabular}

Table 1: $R_{p}$ and $R_{d}$ in $\mathrm{g} \mathrm{cm}^{-4} \mathrm{~s}^{-1}$, and $C$ in $\mathrm{g}^{-1} \mathrm{~cm}^{4} \mathrm{~s}^{2}, \alpha$ in $\mathrm{g}^{-1} \mathrm{~cm} \mathrm{~s}^{2}, \beta$ in $\mathrm{g}^{-1} \mathrm{~cm} \mathrm{~s}$, and $\gamma$ in $\mathrm{g} \mathrm{cm}^{-3}$ $\mathrm{s}^{-1}$.

This example is a typical PA physiological case. In Figure 4 velocity fields are shown during forward peak flow (top) and in presence of backflow (bottom), comparing the 3D-0D, 3D-0D-Stab , 3D-3D and 3D-3D-0D coupling methods. During forward flow, the four approaches lead to very similar velocity fields. In fact, in this configuration the convective stabilization is off, which is verified by the same results obtained for the 3D-0D or 3D-0D-Stab methods. During backflow, the 3D-0D coupling method exhibits perturbations with inward vectors which are very large relative to the flow rate for a few time steps. The 3D-0D-Stab method, while stable, perturbs the hemodynamics by reducing the velocity vectors to almost zero. The 3D-3D approach allows to recover a backflow without instabilities, and similarly for 3D-3D-0D .

In Figure 5, the 3D-0D-Ptot method exhibits velocity vectors with strong spurious radial components at the outlet surface [22]. Spurious in and out of plane vectors can also be seen, even though flow is maximally forward. Moreover, computations are not diverging but lead to a very large in-plane pressure gradient $(\Delta P=26.50 \mathrm{mmHg})$, which is not relevant from a physiological point of view. 

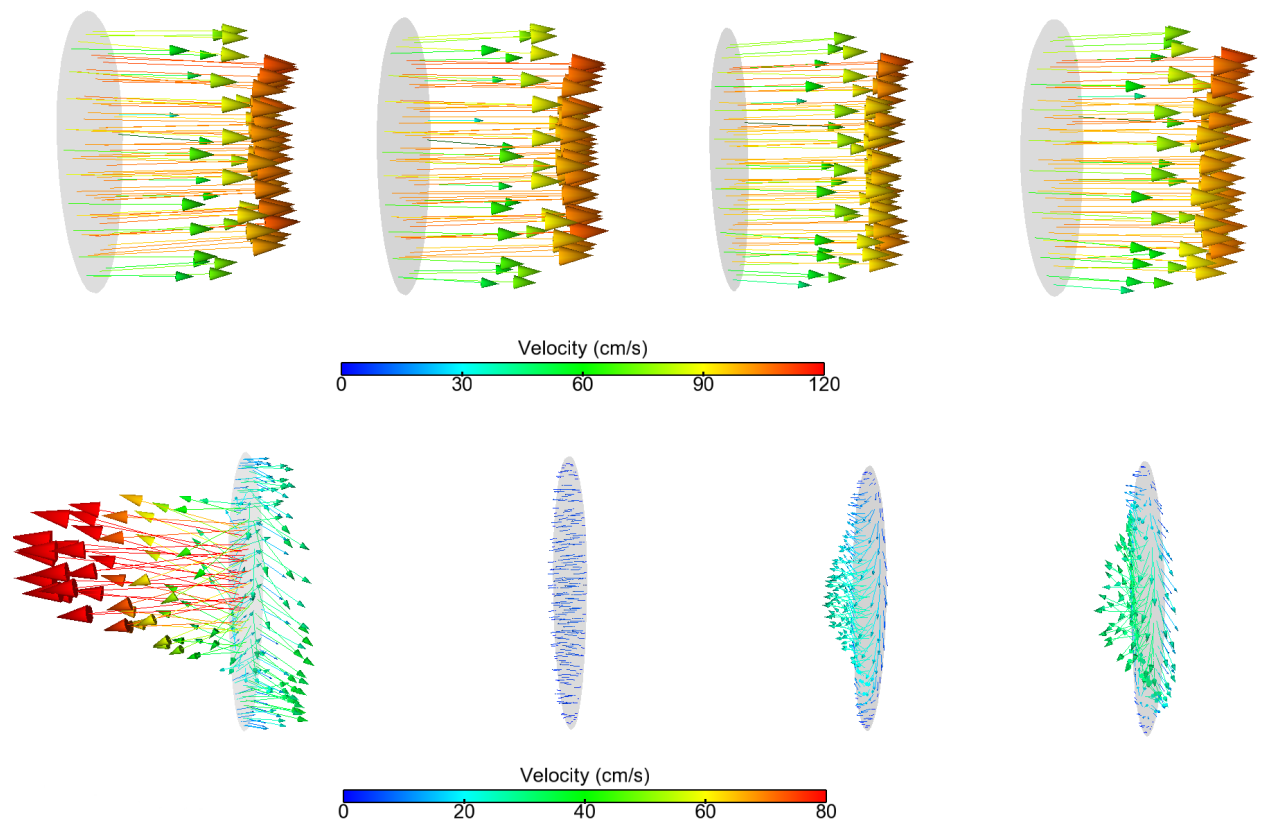

Figure 4: Velocity fields at the LPA interface, when flow at the inlet is maximal forward (top, $\mathrm{t}=0.10 \mathrm{~s}$, $Q_{\max }=378 \mathrm{~cm}^{3} / \mathrm{s}$ ) and is reverse (bottom, $\mathrm{t}=1.06 \mathrm{~s}, Q_{\min }=-66 \mathrm{~cm}^{3} / \mathrm{s}$ ) with the 3D-0D, 3D-0DStab, 3D-3D and 3D-3D-0D coupling methods from left to right.

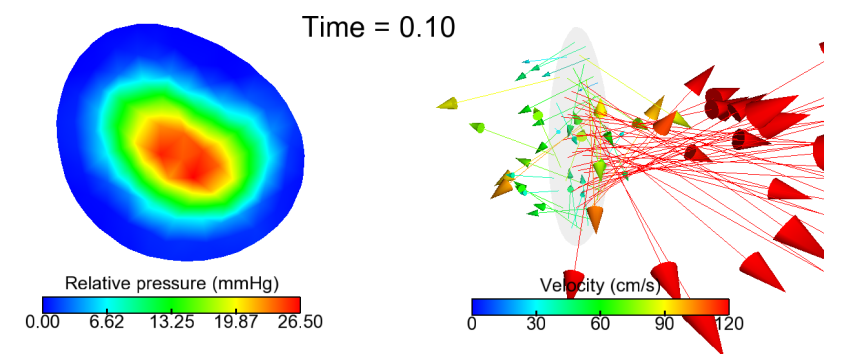

Figure 5: In-plane pressure relative to its minimum value (left) and velocity fields (right) at the LPA interface during maximal forward flow $(\mathrm{t}=0.1 \mathrm{~s})$ with the 3D-0D-Ptot coupling method. 
The time-varying flow rates and average pressure at the coupling surfaces are now analyzed (Figure 6 shows the LPA). For each coupling method, similar flow and pressure results are found on both the left and right-hand sides. Flow and pressure from the 3D-0D-Ptot method are not represented as the obtained values were nonphysiological: although the simulation did not diverge, they were too high with spurious high-frequency tracings. For all other coupling methods, flow varies over time very similarly. Pressure varies over 3 to $20 \mathrm{mmHg}$, in exactly the same way for the 3D-0D and 3D-0D-Stab methods, but a few mmHg lower for the 3D-3D model, while for the 3D-3D-0D model it is closer to the other couplings.

At this point however, it is hard to conclude on which method is best reproducing the real flow behavior, because we do not have access to the three dimensional downstream domain. But this case is highlighting the robustness of the 3D-0DStab and 3D-3D coupling methods to avoid large numerical artefacts due to physiological reverse flow in a patient-specific case.

\subsection{Diseased child pulmonary arteries: a challenging fluid dynamics case}

This patient specific geometry is the first generation of a pulmonary arterial tree of a child affected by a congenital heart disease, the single ventricle pathology (pulmonary atresia with intact ventricular septum). This patient underwent a so-called stage 1 surgical procedure consisting of an anastomosis between the systemic and pulmonary circulatory systems via a $3.5 \mathrm{~mm}$ artificial shunt. For more information about this patient and its model reconstruction see [1].

Figure 7 represents the complete geometrical model (918K tetrahedra, six outlets), the short model with cut pulmonary artery branches (471K tetrahedra, two outlets), and the short model with its artificial parts to the outlet surfaces $(505 \mathrm{~K}$ tetrahedra, 
Flow at left pulmonary arte

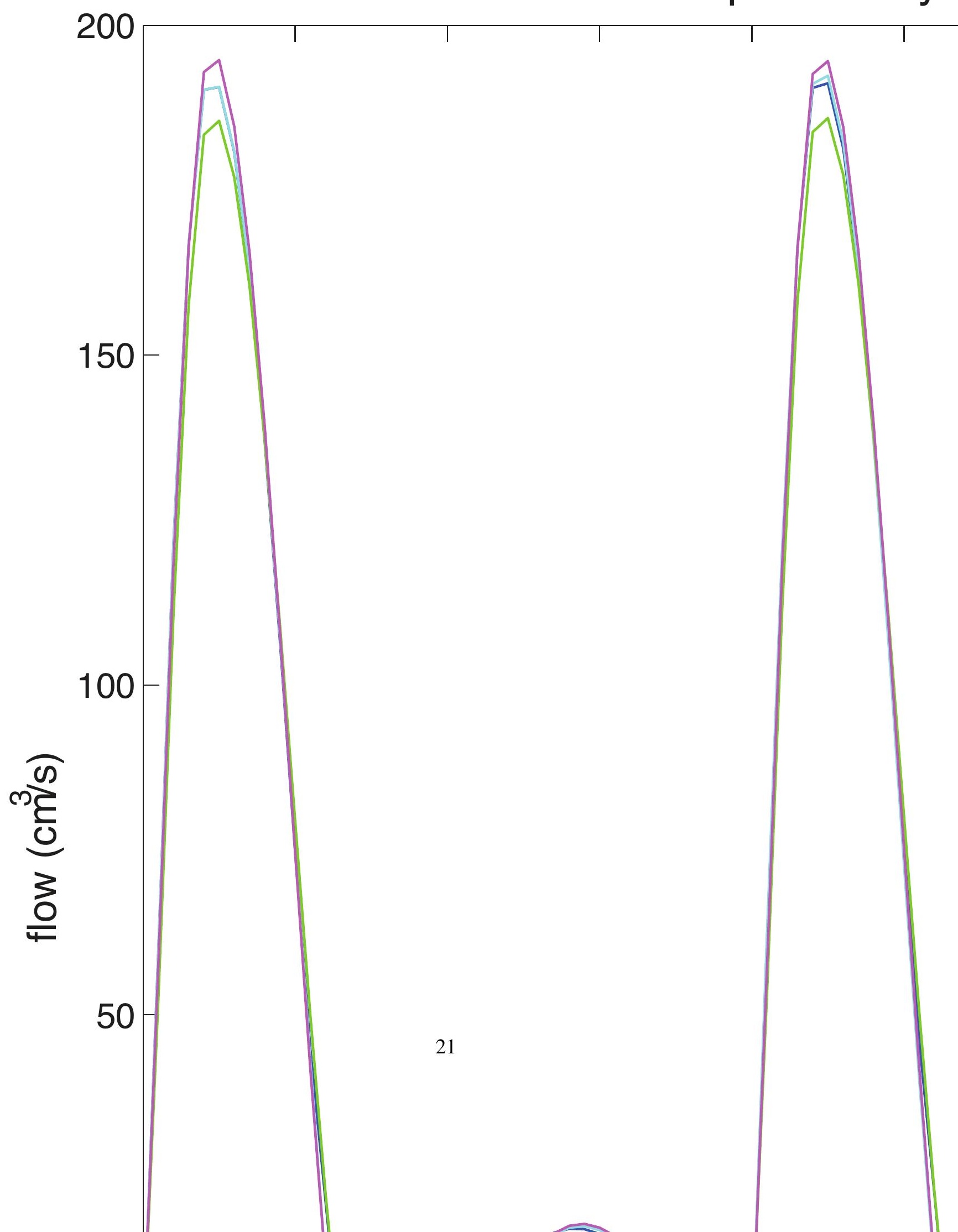




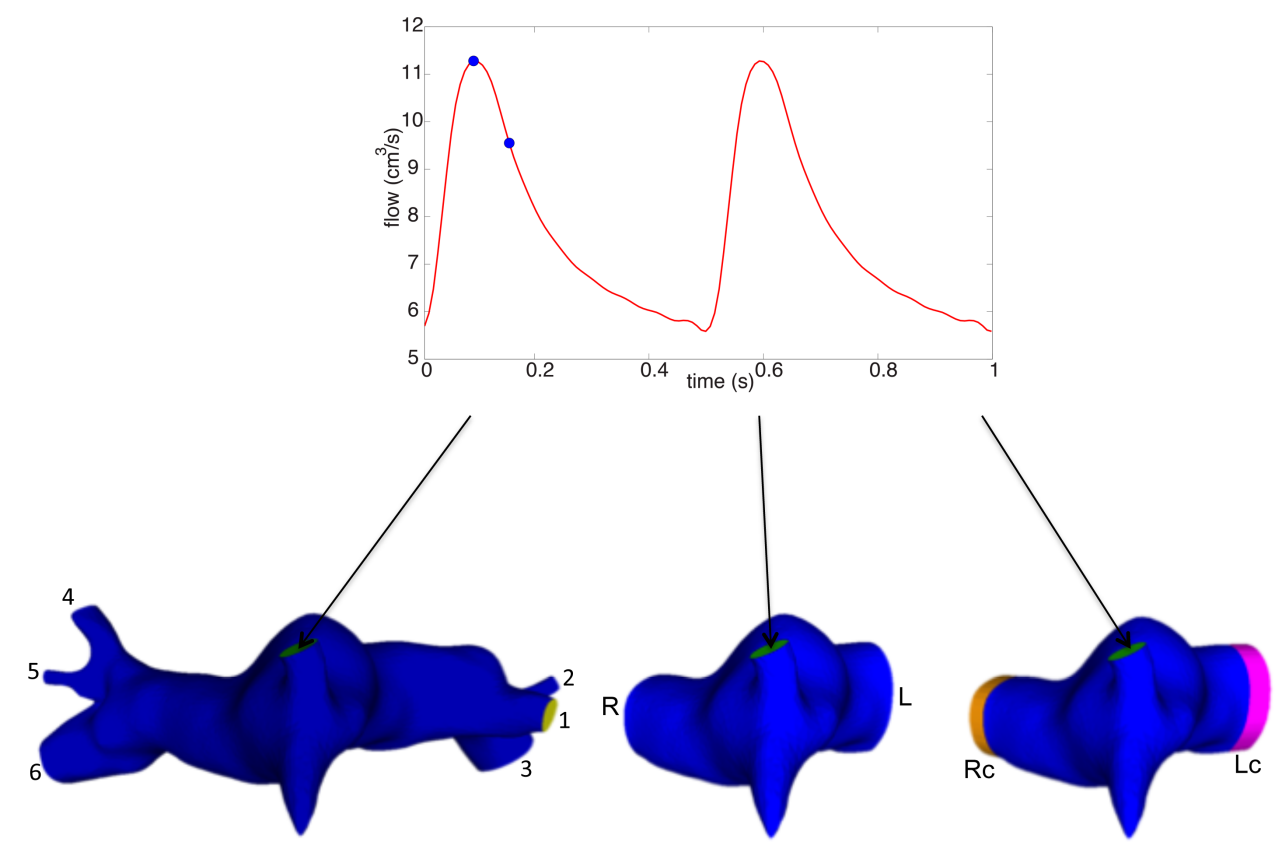

Figure 7: From left to right: complete model (with outlets $1-6$ ), short model (two outlets, R for the RPA and L for the LPA), and short model with the two artificial three dimensional parts on each side, $R_{c}$ and $L_{c}$. Top: imposed inflow shown over two cycles, with the two dots representing maximum flow and decelerating flow respectively. 
2 outlets). As in the previous case, velocity was prescribed at the inlet with a plug profile, following a typical shunt flow tracing. The highest Reynolds number is 3000 . The flow rate varies over one cardiac cycle between $5.8 \mathrm{~cm}^{3} / \mathrm{s}$ and $11.2 \mathrm{~cm}^{3} / \mathrm{s}$ : there is thus no physical flow reversal prescribed at the inlet. The main advantages of this case are first that the effect of artificially cutting the geometry can be assessed, and second that, due to the bifurcation close to the inlet, it is a case of more complex flow than previously.

In Table 2 the values of proximal and distal resistances, and capacitances are given for each of the six outlets of the complete geometrical model. These values were generated to reflect clinical measurements $[1,2]$. A first simulation is run with the 3D-0D coupling approach on the complete geometry. All the coupling methods with the short models are then compared to this reference simulation.

\begin{tabular}{|c|c|c|c||c|c|c|c|}
\hline & 1 & 2 & 3 & 4 & 5 & 6 \\
\hline$R_{p}$ & $3.1910^{1}$ & $4.7310^{1}$ & $6.3810^{0}$ \\
\cline { 2 - 7 } & $4.4010^{-4}$ & $1.7910^{-4}$ & $5.5010^{-3}$ & $1.7110^{1}$ & $4.2610^{1}$ & $5.7510^{0}$ \\
\hline$C$ & $C$ & $1.1410^{-3}$ & $2.3510^{-4}$ & $1.0610^{-2}$ \\
\hline$R_{d}$ & $2.4510^{2}$ & $6.1310^{2}$ & $2.9510^{1}$ & $R_{d}$ & $1.1110^{2}$ & $4.6310^{2}$ & $1.5810^{1}$ \\
\hline
\end{tabular}

Table 2: $R_{p}$ and $R_{d}$ in $\mathrm{g} \mathrm{cm}^{-4} \mathrm{~s}^{-1}$, and $C$ in $\mathrm{g}^{-1} \mathrm{~cm}^{4} \mathrm{~s}^{2}$ for the six Windkessel model outlets of the reference simulation in the complete geometrical model.

In Table 3, the reduced model parameters for the short geometries are given. They were generated from the reference model parameters: at both outlets, an equivalent impedance is computed from the three distal outlet Windkessel model of the complete model. Windkessel model parameters are then optimized to match this impedance. This Windkessel model is then coupled to the 3D Navier-Stokes equations with the 3D-0D, 3D-0D-Stab and 3D-0D-Ptot methods. The 3D-3D parameters in the artificial added parts are displayed, given that the added volumes are such that $V, S$ and $L$ are respectively $0.066 \mathrm{~cm}^{3}, 0.33 \mathrm{~cm}^{2}$, and $0.2 \mathrm{~cm}$ for the left 
side and $0.1 \mathrm{~cm}^{3}, 0.49 \mathrm{~cm}^{2}$, and $0.2 \mathrm{~cm}$ for the right side, reflecting the smaller dimensions of this child anatomy. The 3D-3D-0D parameters are hybrid between the 3D-0D and 3D-3D as described in the beginning of the section.

\begin{tabular}{|c|c|c||c|c|c|}
\hline & $\mathrm{R}$ & $\mathrm{L}$ & & $\mathrm{Rc}$ & $\mathrm{Lc}$ \\
\hline$R_{p}$ & $4.4410^{0}$ & $5.2810^{0}$ & $\gamma$ & $7.1310^{0}$ & $1.2910^{1}$ \\
\hline$C$ & $1.2910^{-2}$ & $6.2010^{-3}$ & $\alpha$ & $1.9610^{-1}$ & $6.3510^{-2}$ \\
\hline$R_{d}$ & $1.3310^{1}$ & $2.5110^{1}$ & $\beta$ & $1.1410^{0}$ & $4.0910^{-1}$ \\
\hline
\end{tabular}

Table 3: Parameters for the different coupling methods in the short models: $R_{p}$ and $R_{d}$ in $\mathrm{g} \mathrm{cm}^{-4} \mathrm{~s}^{-1}$, and $C$ in $\mathrm{g}^{-1} \mathrm{~cm}^{4} \mathrm{~s}^{2}, \alpha$ in $\mathrm{g}^{-1} \mathrm{~cm} \mathrm{~s}^{2}, \beta$ in $\mathrm{g}^{-1} \mathrm{~cm} \mathrm{~s}$, and $\gamma$ in $\mathrm{g} \mathrm{cm}^{-3} \mathrm{~s}^{-1}$.

In Figure 8 velocity fields in the RPA are compared between the different methods at the same location during maximal forward flow (left) and deceleration flow (right). The reference case (top) presents no instability because the flow is smoother at its distal outlets, which is typical of bifurcations quite downstream of complex flow [39]. At maximal forward flow, it shows complex flow in the RPA. This behavior is retrieved on the short model with the 3D-0D coupling method but with inwards velocity vectors. However, the computation is close to divergence. The 3D-0D-Stab coupling method efficiently kills the reverse velocity vectors at the coupling surface so that a similar forward flow motion to the 3D-0D coupling method is retrieved without backflow. Note that the 3D-3D coupling leads to a velocity profile closer to the reference case.

Regarding blood flow behavior during the decelerating phase (right column in Figure 8), there is reverse flow in the reference case and a large proportion of the forward flow is located at the bottom of the surface area. With the 3D-0D coupling method at outlet surfaces of the cut model, the computation is diverging, but the 3D-0D-Stab coupling method leads to a blood flow behavior where velocity vectors are underestimated at the center and top of the coupling surface area. In 

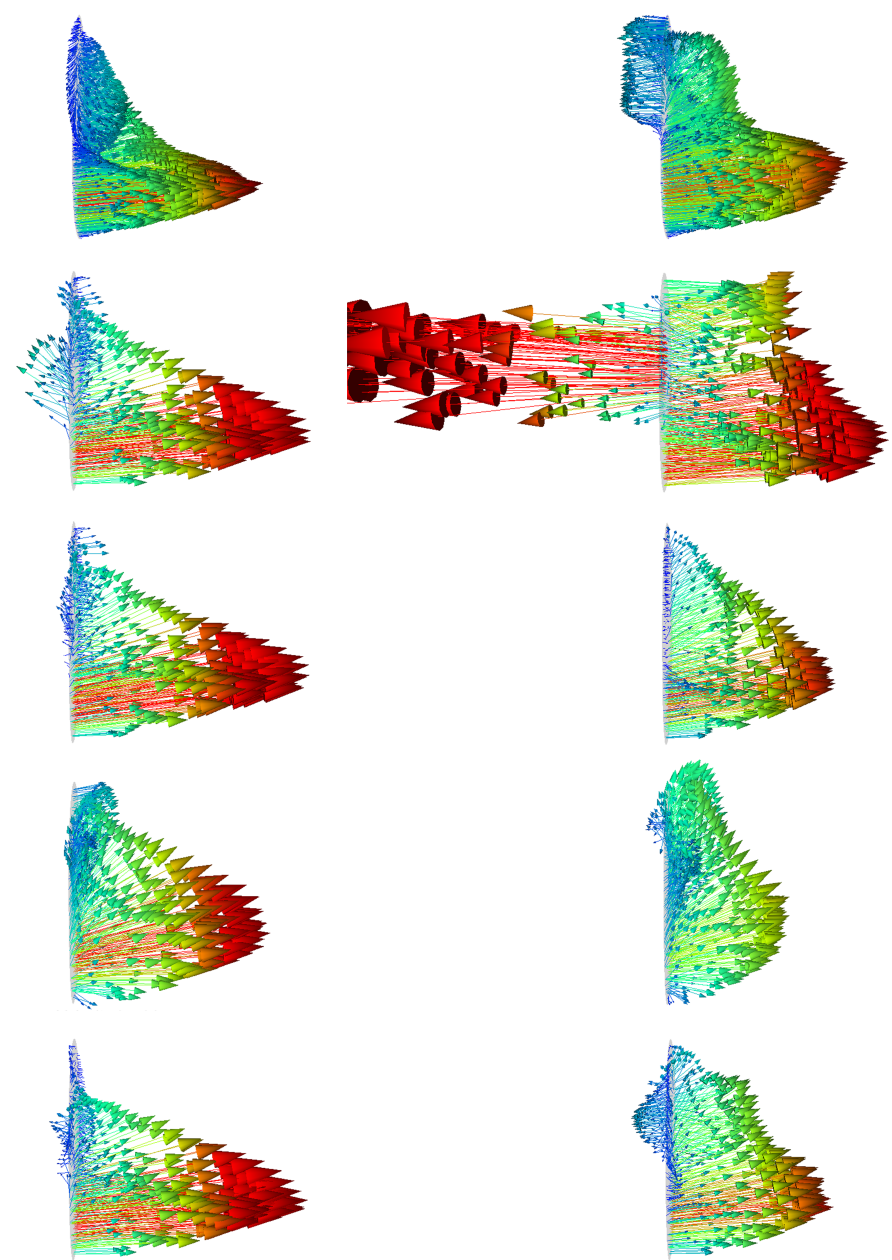

Figure 8: Velocity fields in RPA at peak inlet flow (left, $\mathrm{t}=0.0936 \mathrm{~s}, Q_{\max }=11.2 \mathrm{~cm}^{3} / \mathrm{s}$ ) and during decelerating flow (right, $t=0.156 \mathrm{~s}$ ) comparing the reference, 3D-0D, 3D-0D-Stab, 3D-3D and 3D3D-0D coupling methods from top to bottom. The two times are shown as dots on the inflow Figure 7. Color encodes velocity magnitude from 0 (blue) to $90 \mathrm{~cm} / \mathrm{s}$ (red). 
the 3D-3D coupling approach, flow motion is more homogeneous in terms of size and direction of the velocity vectors. The 3D-3D-0D coupling method leads during maximal forward flow to a velocity field close to the coupling of reduced model with the 3D-0D-Stab method. During deceleration the majority of flow is located at the bottom of the coupling surface and backflow is authorized in the upper part.

The obtained flow behavior is thus close to the reference case.

The velocity field for the 3D-0D-Ptot coupling method behaves as in the previous patient-specific case of adult pulmonary arteries. However, the computation is diverging.

In the figures above, the RPA side is presented. In fact, the LPA is turning in the downstream 3D domain of the reference case in such a way that hemodynamics are even more different between the reference and the cut models than for the RPA. Next, the resulting flow rates are compared at coupling surfaces (Figure 9 shows as an example the RPA), between the 3D-0D , 3D-0D-Stab , 3D-3D and 3D-3D-0D coupling models. In all cases, most of the flow goes to the RPA, following a dynamics close to that of the inlet flow, with a peak in systole followed by a rapid decay. The flow rates oscillate in time in diastole due to the complex flow structures created by the interaction of the impinging inflow and the patientspecific geometry. However, for the 3D-0D case the flow rates largely oscillate before the simulation diverges. In terms of magnitude, the coupling method that is closer to the reference case is the 3D-0D-Stab method, on both sides. The 3D-3D coupling approach is leading to a satisfying blood flow behavior but changes the distribution of flow between the left and right-hand sides for this choice of parameters: it slightly overestimates flow in the RPA and thus underestimates flow in the LPA. However, the hybrid coupling method 3D-3D-0D is close to the reference case. The diverging 3D-0D-Ptot results are not represented because the simulation 
diverged early on $(\mathrm{t}=0.0936 \mathrm{~s})$.

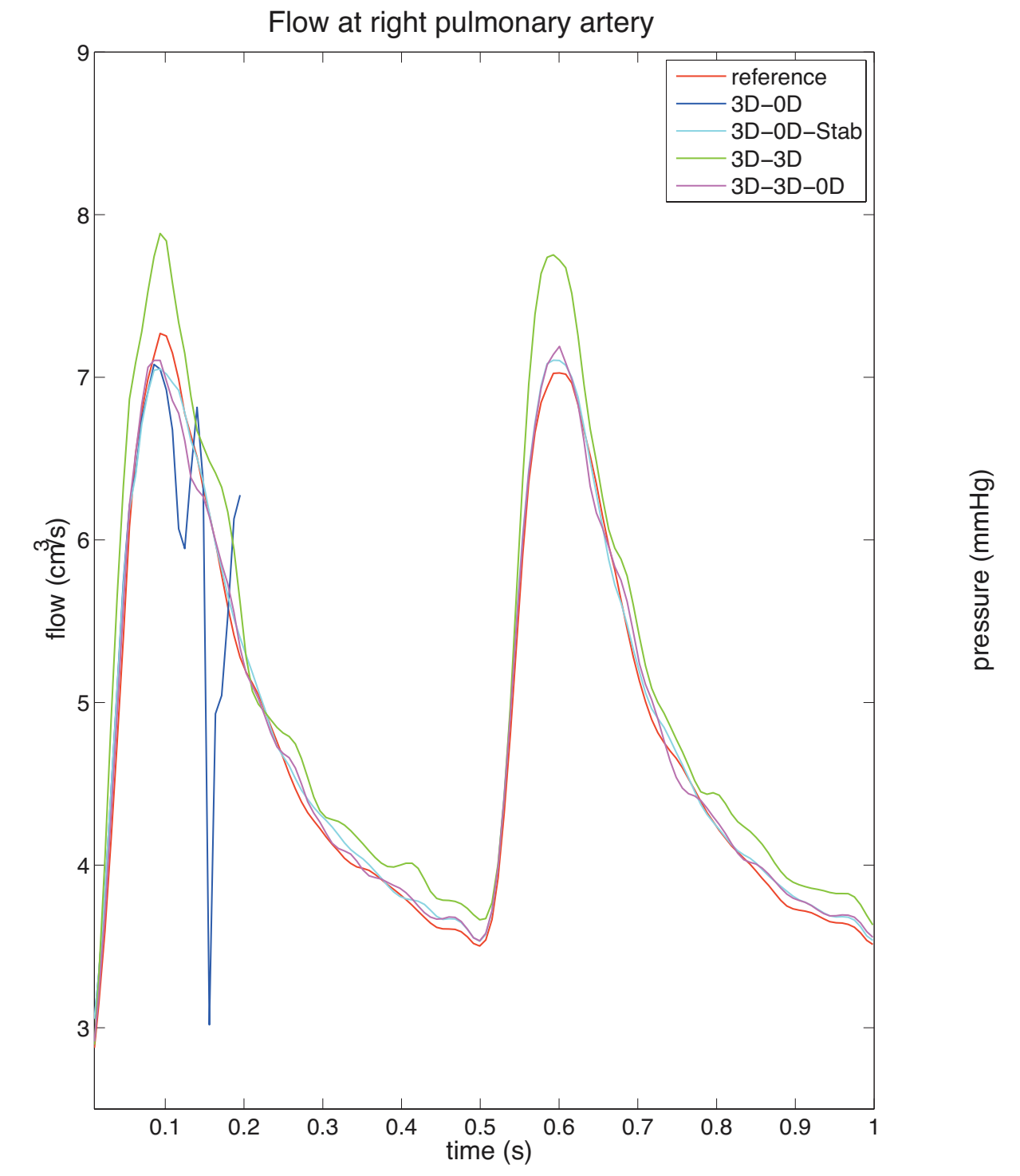

Figure 9: Flow rate (left) and pressure (right) over two cardiac cycles in the RPA for the different coupling methods. 
Lastly, Figure 9 shows the corresponding pressure waveforms. The time dynamics are similar for all cases and on both sides, except for the 3D-0D case which diverges in the first cardiac cycle. After the transitory first cycle, the second cycle is closer to a periodic solution with a sharp rise in systole, followed by a smooth diastole decay. The closest results to the reference case is the 3D-0D-Stab method for both sides. The 3D-3D approach underestimates pressure for both sides. However the hybrid 3D-3D-0D coupling approach more closely reproduces the reference results.

To summarize this case, both 3D-0D and 3D-0D-Ptot couplings lead to divergence of the simulation, although there is negative flow rate neither at the inlet nor at the outlets. This is highlighting the need for robust numerical methods in the presence of complex flow, such as the 3D-0D-Stab and 3D-3D or 3D-3D-0D coupling methods. The three presented similarities and differences with the reference case, which is the ground truth, 3D-3D-0D being the best compromise in this example.

\subsection{Adult patient-specific aorta: complex flow generated during deceleration}

This last patient-specific case is an abdominal adult aorta. Figure 10 consists of the inlet flow rate over time and the three tested geometrical models: the complete 3D model containing $608 \mathrm{~K}$ tetrahedra and 9 outlet surfaces, the short model containing $473 \mathrm{~K}$ tetrahedra and 6 outlet surfaces where the abdominal aorta was cut to remove the iliac branches, and the same cut model but with an artificial 3D part at its descending aorta outlet, containing $499 \mathrm{~K}$ tetrahedra. Velocity is prescribed at the inlet of the model, as a plug profile following the flow tracing of Figure 10. As is typical in the supra celiac descending aorta, this flow rate does not contain reverse flow over the entire cardiac cycle. The maximal flow is $Q_{\max }=177 \mathrm{~cm}^{3} / \mathrm{s}$ 
442

and the minimal flow is $Q_{\min }=7 \mathrm{~cm}^{3} / \mathrm{s}$. Deceleration is large enough to generate complex flow behavior in all the geometrical models regardless of the outlets coupling methodology at hand. The complete model is the reference where three dimensional Navier-Stokes equations are coupled to reduced models at every outlet with the 3D-0D coupling method.

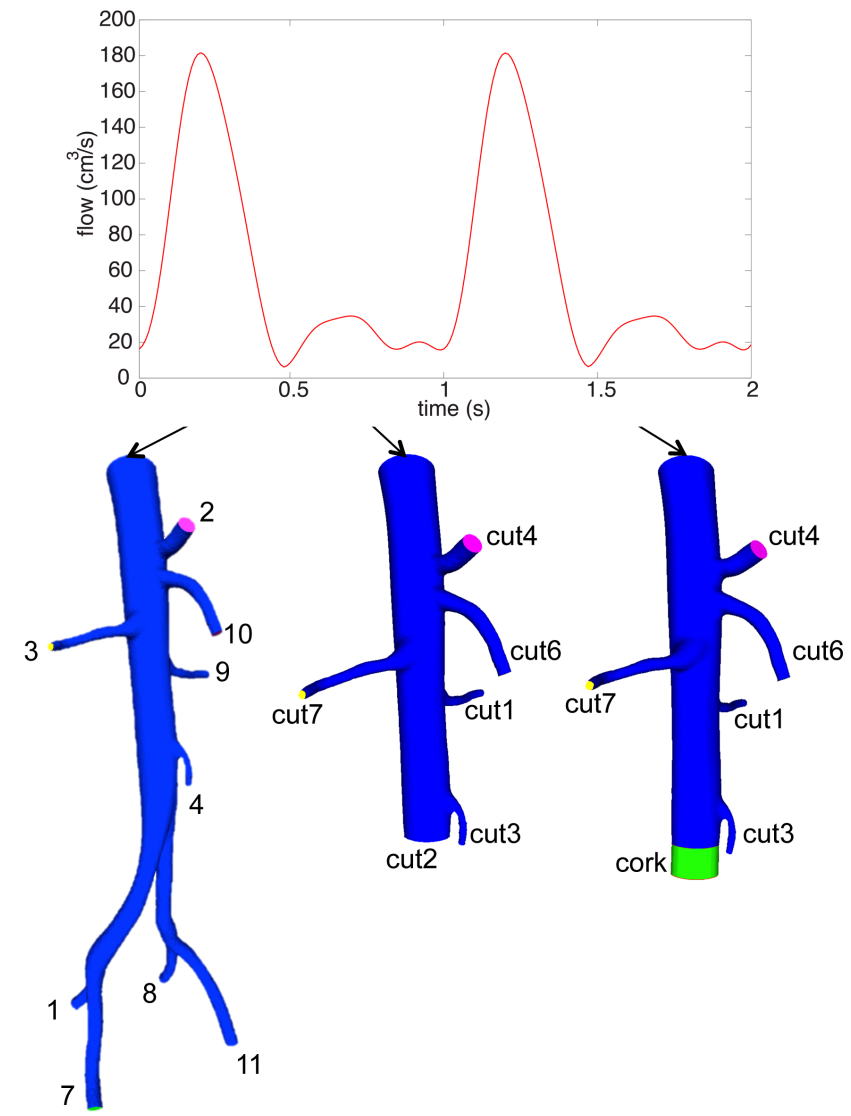

Figure 10: Top: imposed inflow $\left(\mathrm{cm}^{3} / \mathrm{s}\right)$ shown over two cycles. Below, from left to right: the complete 3D model, the cut 3D model and the cut 3D model with its artificial 3D part tagged "cork". The outlets are all labeled for future reference.

443 Table 4 contains the values of the proximal and distal resistances, and capacitances 
for each of the nine outlets of the complete geometrical model. These values have been set to match clinical measurements [41].

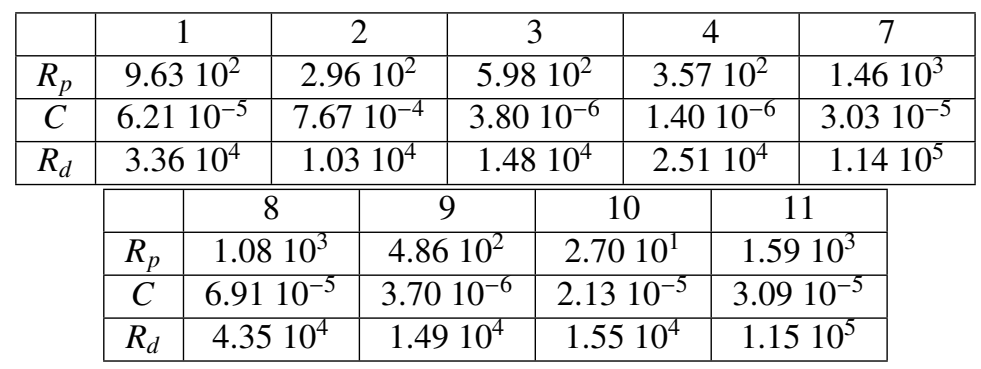

Table 4: Reduced model parameters for each outlet of the reference model, as labeled in the full model of Figure 10. $R_{p}$ and $R_{d}$ in $\mathrm{g} \mathrm{cm}^{-4} \mathrm{~s}^{-1}$, and $C$ in $\mathrm{g}^{-1} \mathrm{~cm}^{4} \mathrm{~s}^{2}$.

Table 5 contains values of proximal and distal resistances, and capacitances for each of the six outlets of the cut geometrical model. These reduced model parameters for the cut abdominal outlet surface (cut2) were not generated from the ones of the four outlet surfaces of the reference model (labeled 1,7, 8 and 11) as was done in the previous test case, as this was leading to too inaccurate results. Instead, the three Windkessel model parameters of this cut outlet were identified by a Kalman filtering approach based on the flow and pressure tracings from the reference simulation $[34,35]$.

\begin{tabular}{|c|c|c|c|c|c|c|}
\hline & cut1 & cut2 & cut3 & cut4 & cut6 & cut7 \\
\hline$R_{p}$ & $4.8610^{2}$ & $8.2110^{2}$ & $3.5710^{2}$ & $2.9610^{2}$ & $2.7010^{1}$ & $5.9810^{2}$ \\
\hline$C$ & $3.7010^{-6}$ & $2.0510^{-4}$ & $1.4010^{-6}$ & $7.6710^{-4}$ & $2.1310^{-5}$ & $3.8010^{-4}$ \\
\hline$R_{d}$ & $1.4910^{4}$ & $2.1310^{4}$ & $2.5110^{4}$ & $1.0310^{4}$ & $1.5510^{4}$ & $1.4810^{4}$ \\
\hline
\end{tabular}

Table 5: Reduced model parameters for each of the six outlet of the cut model (for the 3D-0D and 3D-0D-Stab coupling methods), as labeled in Figure 10. $R_{p}$ and $R_{d}$ in $\mathrm{g} \mathrm{cm}^{-4} \mathrm{~s}^{-1}$, and $C$ in $\mathrm{g}^{-1} \mathrm{~cm}^{4}$ $\mathrm{s}^{2}$.

Table 6 contains values of proximal and distal resistances, and capacitances for each of the five outlets of the original part and coefficient $\alpha, \beta$ and $\gamma$ for the 3D-3D model in the artificial part. The latter were chosen given that the added volumes 
are such that $V, S$ and $L$ are respectively $1.8 \mathrm{~cm}^{3}, 1.8 \mathrm{~cm}^{2}$, and $1 \mathrm{~cm}$. For the 3D-3D-0D model, the values are the same, except for the artificial part for which changes are described at the beginning of the results section.

\begin{tabular}{|c|c|c|c|c|c||c|c|}
\hline & cut1 & cut3 & cut4 & cut6 & cut7 & & cork \\
\hline$R_{p}$ & $4.8610^{2}$ & $3.5710^{2}$ & $2.9610^{2}$ & $2.7010^{1}$ & $5.9810^{2}$ & $\gamma$ & $1.4910^{3}$ \\
\hline$C$ & $3.7010^{-6}$ & $1.4010^{-6}$ & $7.6710^{-4}$ & $2.1310^{-5}$ & $3.8010^{-6}$ & $\alpha$ & $1.1310^{-4}$ \\
\hline$R_{d}$ & $1.4910^{4}$ & $2.5110^{4}$ & $1.0310^{4}$ & $1.5510^{4}$ & $1.4810^{4}$ & $\beta$ & $2.6010^{-5}$ \\
\hline
\end{tabular}

Table 6: Reduced model parameters for each outlet of the 3D-3D coupling model, as labeled in the cut model with "cork" in Figure 10. $R_{p}$ and $R_{d}$ in $\mathrm{g} \mathrm{cm}^{-4} \mathrm{~s}^{-1}$, and $C$ in $\mathrm{g}^{-1} \mathrm{~cm}^{4} \mathrm{~s}^{2}, \alpha$ in $\mathrm{g}^{-1} \mathrm{~cm} \mathrm{~s}^{2}, \beta$ in $\mathrm{g}^{-1} \mathrm{~cm} \mathrm{~s}$, and $\gamma$ in $\mathrm{g} \mathrm{cm}^{-3} \mathrm{~s}^{-1}$.

Hemodynamics are compared through the cut abdominal aortic surface of the reference simulation with the abdominal aortic outlet of the other models. On the left column of Figure 11, i.e. during forward flow, the velocity field behavior is closer to the reference for the 3D-0D coupling and thus also for 3D-0D-Stab method since the vectors are all pointing outwards. The 3D-3D approach leads to a flatter velocity profile. 3D-3D-0D is in between these two methods. However, during reverse flow, the reference case concentrates the higher velocity magnitudes on the exterior part of the surface area. The 3D-0D coupling method results in high central velocity vectors, eventually leading to divergence of the simulation. This time, the 3D-0D-Stab and 3D-3D-0D coupling exhibit some backflow but with a homogeneous distribution of velocity vectors on the surface area. The 3D-3D model is similar, but with velocity magnitude closer to the average reference case.

Figure 12 shows the Ptot coupling method results, with spurious radial velocity vectors, exhibiting here particularly the well-known effect of the total pressure coupling [22]. In this case the simulation actually diverges. 

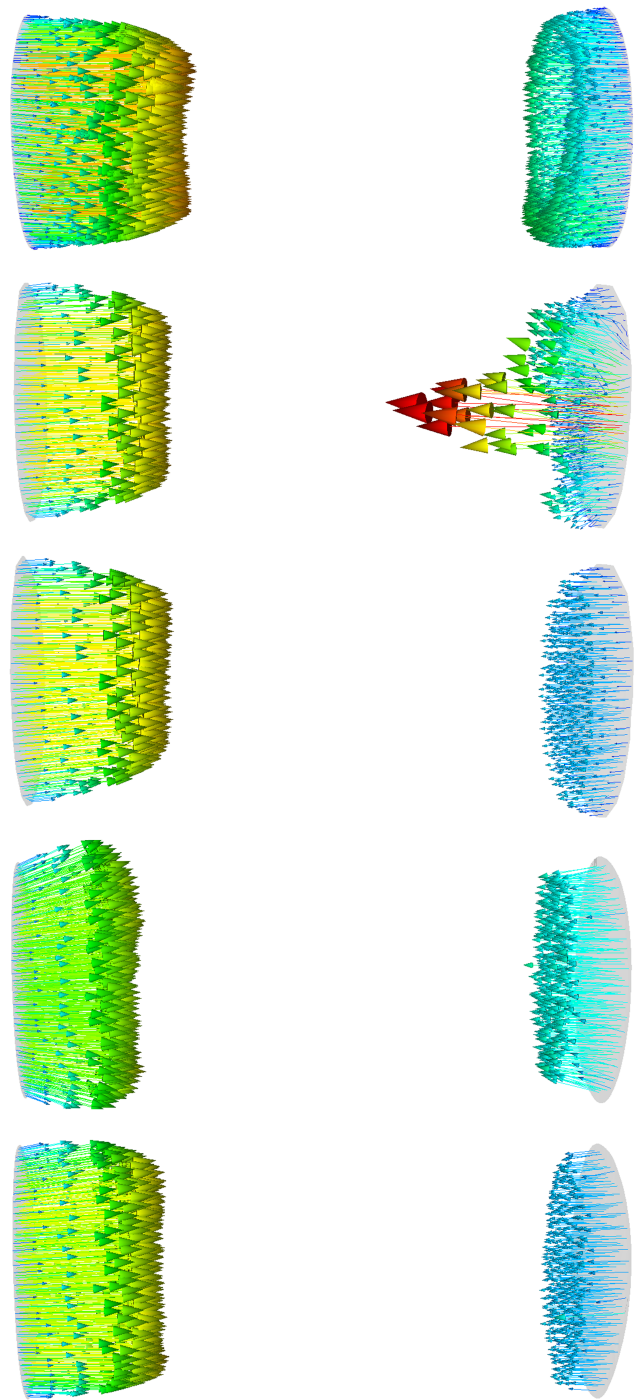

Figure 11: Velocity fields at two different times, $t=0.21 \mathrm{~s}$ (maximal forward inlet flow, left) and $\mathrm{t}=0.46 \mathrm{~s}$ (maximal reverse flow at outlet, right) for from top to bottom: reference, 3D-0D, 3D-0DStab , 3D-3D and 3D-3D-0D coupling models. Color encodes for velocity magnitude from 0 (blue) to $50 \mathrm{~cm} / \mathrm{s}$ (red). 


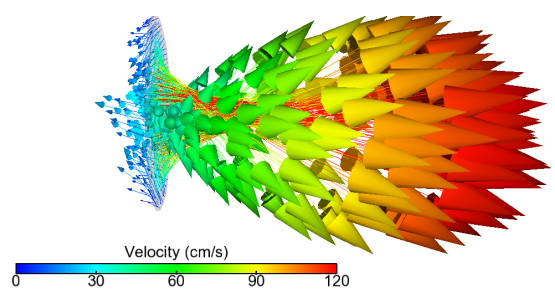

Figure 12: Velocity fields during maximal forward flow with the 3D-0D-Ptot coupling model.

Next, in Figure 13, the resulting time-varying flow rates are compared at the same surface, between the 3D-0D , 3D-0D-Stab , 3D-3D and 3D-3D-0D coupling models. All cases follow a dynamics with a peak in systole followed by a rapid deceleration with significant flow reversal and then forward flow again in the last part of diastole. The curves are neither completely synchronized in time nor matching completely in amplitudes. This time, for the 3D-0D case the flow rate does not largely oscillate before the simulation diverges. Moreover the 3D-0D-Stab coupling method is disturbing the blood flow behavior, with a flow rate amplitude lower than the reference one, where maximum flow is lower and minimum flow is higher than reference flow rate. The 3D-3D follows closely. The coupling method that reproduces as best as possible the flow tracing from the reference case is the 3D-3D-0D coupling method even if the minimum flow is overestimated.

In Figure 14, the corresponding pressure curves are compared: although they all follow the same typical pressure dynamics of a peak in systole followed by a smooth decay in diastole, all coupling methods overestimate the reference pressure. They are all shifted in time differently than for the flow rates. The 3D-0D case overestimates pressure the least, but it diverges in diastole. For this test case, the 3D-0D-Stab method is the furthest from the reference case whereas the 3D3D-0D coupling model is the closest. The 3D-3D coupling approach is in between in terms of magnitude, but with an additional time shift. 


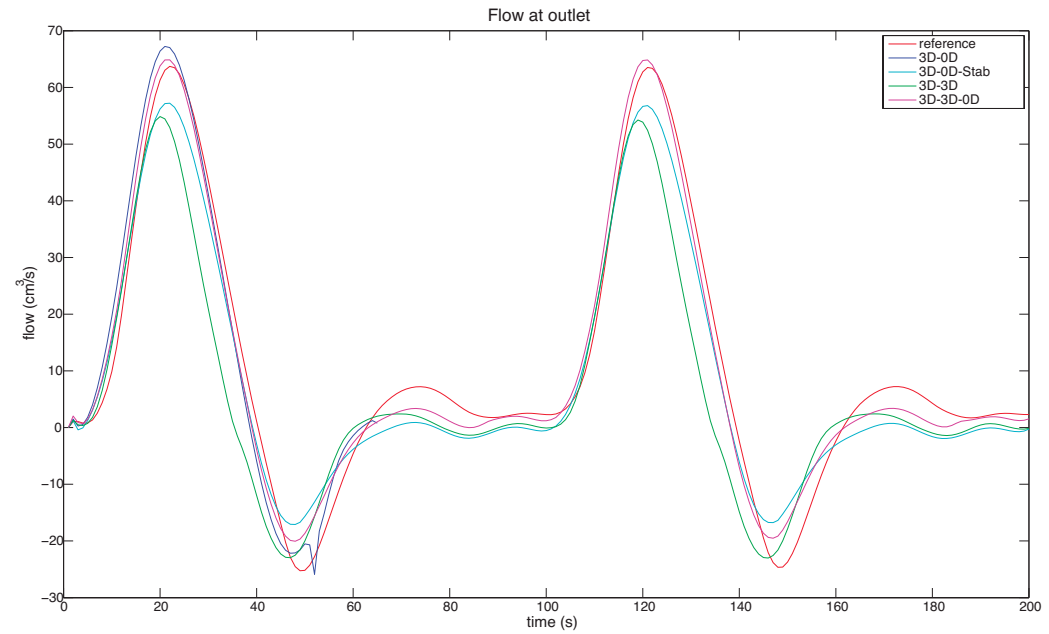

Figure 13: Flow rates over two cardiac cycles at the infrarenal (cut2) coupling interface, with the different coupling methods.

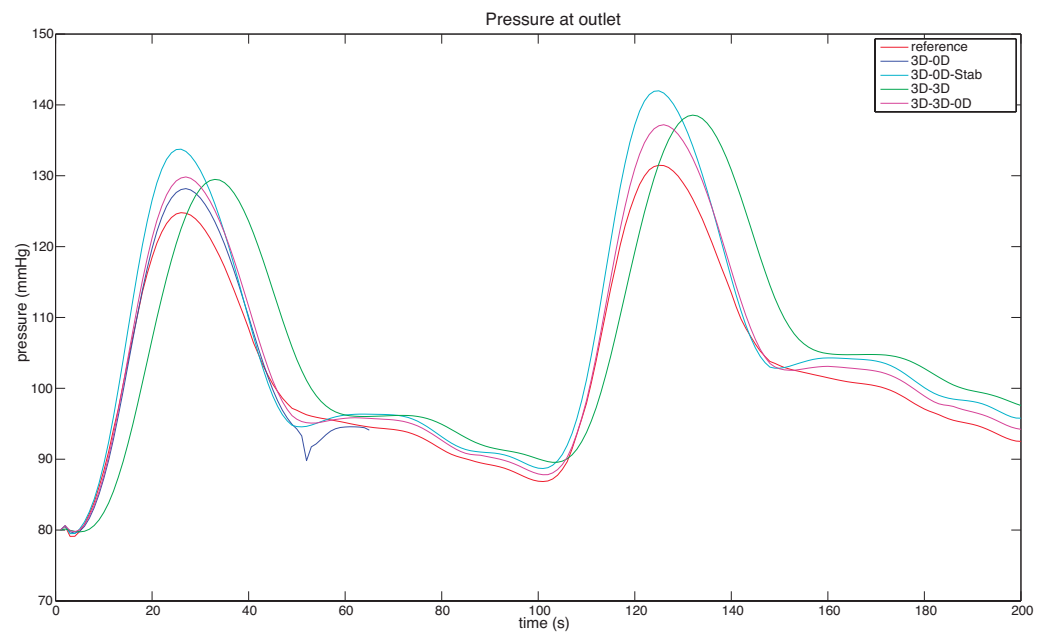

Figure 14: Surface-averaged pressure over two cardiac cycles at the infrarenal (cut2) coupling interface with the different coupling methods. 
In summary for this case, one can observe that both 3D-0D and 3D-0D-Ptot couplings lead to divergence of the simulation, due to the negative flow rate at the outlet, although the flow is entering at the inlet. Such a flow rate difference is typical of these abdominal aorta locations [41]. In all the converging cases during back flow, the velocity vectors are all aligned in the same inward direction. The 3D-0D-Stab , 3D-3D and 3D-3D-0D coupling methods presented similarities and differences with the reference case, which is the ground truth, 3D-3D-0D being again the best compromise in this example.

\section{Discussion and Conclusion}

\subsection{Divergence behavior}

Different existing methods have been implemented and compared to couple threedimensional finite element Navier-Stokes simulations to lumped parameter models, and in particular to the three-element Windkessel model. Differences in numerical implementations that might also affect numerical stability (e.g [31] and references therein) are not discussed here, but rather coupling approaches at the continuous level. The first (standard) idea has been to impose weakly that at the coupling interface the normal component of the stress tensor is equal to a surface-homogeneous pressure computed from the reduced model. Such a coupling was shown above to lead to an (uncontrolled) convective boundary energy term, potentially destabilizing with flow reversal at the interface. In presence of complex flow at that location, simulations are diverging in the patient-specific examples presented above. This behavior is consistent with other examples from the literature $[1,40]$. Note that for the two last patient-specific cases, although the same 3D-0D coupling was imposed in the full 3D-models, the simulations did not diverge: as the geometry branches off, flow gets more streamlined as observed in $[39,40]$. To get rid of this poten- 
tially destabilizing boundary convective term, the 3D-0D-Ptot coupling method was also implemented. This coupling approach was considered for example in $[5,22]$. Similarly to what [22] observed already for stationary flow in a pipe, abnormally large radial components of the velocity vectors at the coupling surface were observed during forward flow. Likely because of higher Reynolds numbers and more complex flow in these patient-specific cases, spurious peripheral inward and out of plane velocity vectors were seen, even in case of forward flow. This generated abnormally large in-plane pressure gradient and eventually lead to divergence of the simulations when forward flow became higher.

\subsection{About the convective stabilisation}

These two methods highlighted the sensitivity of the simulation stability to the choice of coupling method. It is not a priori possible to predict which simulation will be stable throughout or diverge. Since the first coupling only diverges typically with some inward and non-zero tangential velocity vector or complete back flow at the coupling interface, natural ideas are to enforce the tangential component to be zero or to even constrain the whole velocity profile. As these have been shown to be non-ideal [29], here the convective 3D-0D-Stab method was retained. Recall that it only acts when velocity vectors point inwards at the coupling interface. It was introduced in [4] for cardiovascular applications with an energetically over-stabilizing effect as discussed above, and successfully tested with a smaller energy-dissipative coefficient (a value of $\theta$ of 0.5 or less was found enough to ensure stability) in $[29,31,32]$ in cardiovascular and respiratory contexts. In these papers, without this stabilization the simulations would have diverged. Here the version that exactly annihilates the energetically destabilizing convective term was implemented. It leads in fact to a similar stabilizing behavior. The present re- 
sults highlight the robustness of this method under different conditions of complex and back flows. However such a coupling involves enforcing an ad-hoc inhomogeneous normal stress over the interface area and in practice generally induces an over-killing of the inward velocity vectors. Besides, as noted by [36], this leads to a weak formulation that is not consistent with the original strong boundary condition of the reduced model coupling. A consistent formulation (with a value of $\theta$ of one) has been proposed by [20] in respiratory mechanics (see this reference for earlier references about imposition of total momentum flux) but it requires to also prescribe the convective term and thus to know the velocity vectors at the coupling boundary or to make some further assumption on the velocity profile at the boundary [20,23]. The convective stabilization with $\theta=1$ resulted in single tube cases in a flatter velocity profile than in their total momentum flux case. It is interesting to note that in respiratory applications for which the back flow is very significant (whole expiration phase), the necessity to stabilize the convective term one way or another was found crucial $[23,26,32]$.

\subsection{Discussion on the modified Navier-Stokes method, its advantages and limits}

One of our main aims was thus to develop a new three-dimensional finite element coupling method where reduced model parameters were three-dimensionally distributed in a modified Navier-Stokes equation into a small outer portion. The goal was to obtain a blood flow behavior as close as possible to a 3D-0D coupling into an extended geometrical model with more branches. From a theoretical point of view, this approach has the advantage that it does not necessitate a coupling condition at the interface: it thus does not enforce the normal component of the stress to be homogeneous as in the 3D-0D method or inhomogeneous in an ad-hoc way as in the 3D-0D-Stab method. Another advantage is that it generates an energy balance 
that is similar to the 3D-0D method, but, if the distal boundary of the modified part is set to zero velocity (as was done in the 3D-3D simulations), it does not include the potentially destabilizing convective boundary term. If this distal velocity is not zero (as was the case in the 3D-3D-0D simulations), the velocity field is damped and smoothed by the modified portion before it reaches the boundary, so it does not develop instabilities in practice.

Numerically, as a general trend, the results suggest that the 3D-0D-Stab coupling method is more accurately reproducing forward flow whereas during deceleration or reverse flow it is less invasive to use the three-dimensional coupling 3D-3D approach than the robust 3D-0D-Stab model. An excellent compromise was reached by the 3D-3D-0D method, a hybrid method which regularizes the flow in an artificial portion, with a small dissipative term, representing a small fraction of the proximal resistance. It effectively stabilized the simulations, without significantly affecting forward flow but allowing more freely inward velocity vectors than the 3D-0D-Stab method. Regarding pressure and flow tracings at the coupling interfaces of the three patient-specific cases, all coupling methods could reproduce the general temporal dynamics, unless they diverged. However, quantitative pressure and flow differences could be observed. In fact, in biomedical applications, the existing coupling methods and the ones developed in this article all involve tuning of the distal parameters in order to be as coherent as possible to clinical hemodynamics measurements [43]. Such tuning was not the focus of this work, and is matter of intense recent research (e.g [1, 7, 13, 24, 34, 35, 38, 39]). Here, when the reference model (extended model with Windkessel reduced models at its ends) was available, it was quite simple to deduce or automatically tune the corresponding Windkessel model at the coupling interface of the cut model. This made sure that the 3D-0D and 3D-0D-Stab results were close to the reference case. However, such equivalence or tuning was less obvious to do for the new 3D-3D parameters $(\alpha, \beta$ and 
$\gamma$ ): a better match of the pressure and flow waveforms could be achieved by manually tuning these parameters, but we thought it would make more sense to present the results with the most straightforward parameterization. Actually in general the reference model would not be available (otherwise it would be used without cutting) and the modified Navier-Stokes parameters (in its original or hybrid version) would be tuned in order to match clinical hemodynamics measurements instead of matching reduced model parameters $R_{p}, C$ and $R_{d}$. This approach is not developed at this time and could be part of future work. Another important question that was not addressed in detail here is the length of the artificial domain. Size should not of course be too large to avoid unnecessary additional computational cost. If it is too small, pressure and velocity change too abruptly (3D-3D) or the velocity field is not regularized enough before hitting the outlet (3D-3D-0D). We have not carried out an extensive sensitivity analysis on the subject, but we noticed that multiplying the cork volume of the presented results by a factor two did not affect the numerical stability of the solution.

\subsection{Comparison between cut and reference models}

Interestingly, several methods lead to more similar results between each other than compared to the non-cut reference model. This is particularly true when the downstream part is geometrically different than the upstream, as was the case of the LPA in the child pulmonary example. The distribution of flow rates among the different downstream vessels can also affect the flow in the non-cut upstream region. This highlights the importance of including enough downstream geometry to not affect the area of interest in the three-dimensional part in biomedical applications $[1,39]$. 


\subsection{Possible extension of the work}

Finally, this 3D-3D method could be extended to a more extensive lumped parameter model through the $\beta$ term. The latter could include a distal pressure, representing the link with the rest of the reduced model. Furthermore, it would be interesting to implement a space-varying $\gamma$ parameter. This dissipative term in the Navier-Stokes equation is known to regularize the velocity profile to a plug-like profile (like at the interface with a porous medium), which helps here to stabilize the simulation but is also responsible for a significant impact on the velocity field at the interface. The coupling approach would be less invasive in particular during forward flow if this parameter was changing more smoothly. This is probably why the 3D-3D-0D was found to be the best compromise between robustness and non-invasiveness.

Acknowledgments. This work was supported by the Leducq Foundation as part of the Transatlantic Network of Excellence for Cardiovascular Research "Multi-scale modeling of single ventricle hearts for clinical decision support", and Associated team Cardio INRIA grant.

\section{References:}

[1] Arbia, G., Corsini, C., Baker, C., Pennati, G., Hsia, T.Y., Vignon-Clementel, I.E., for MOCHA: Pulmonary hemodynamics simulations before stage 2 single ventricle surgery: patient-specific parameter identification and clinical data assessment. Cardiovascular Engineering and Technology 6(3), 268-280 (2015). DOI 10.1007/s13239-015-0212-3

[2] Arbia, G., Corsini, C., Esmaily Moghadam, M., Marsden, A.L., Migliavacca, F., Pennati, G., Hsia, T.Y., Vignon-Clementel, I.E., for MOCHA: Numerical 
blood flow simulation in surgical corrections: what do we need for an accurate analysis? Journal of Surgical Research 186(1), 44-55 (2014)

[3] Baretta, A., Corsini, C., Marsden, A.L., Vignon-Clementel, I.E., Hsia, T.Y., Dubini, G., Migliavacca, F., Pennati, G., for MOCHA: Respiratory effects on hemodynamics in patient-specific CFD models of the Fontan circulation under exercise conditions. European Journal of Mechanics B-Fluids 35, 6169 (2012). DOI 10.1016/j.euromechflu.2012.01.012. URL <GotoISI> : // WOS : 000306039700010

[4] Bazilevs, Y., Gohean, J.R., Hughes, T.J.R., Moser, R.D., Zhang, Y.: Patientspecific isogeometric fluid-structure interaction analysis of thoracic aortic blood flow due to implantation of the Jarvik 2000 left ventricular assist device. Computer Methods in Applied Mechanics and Engineering 198(4546), 3534-3550 (2009). DOI 10.1016/j.cma.2009.04.015. URL <GotoISI> : //WOS : 000270702500004

[5] Begue, C., Conca, C., Murat, F., Pironneau, O.: Les équations de Stokes et de Navier-Stokes avec des conditions aux limites sur la pression. Seminaire College de France (1988)

[6] Bertoglio, C., Caiazzo, A.: A tangential regularization method for backflow stabilization in hemodynamics. Journal of Computational Physics 261, 162$171(2014)$

[7] Bertoglio, C., Moireau, P., Gerbeau, J.F.: Sequential parameter estimation for fluid-structure problems: Application to hemodynamics. International Journal for Numerical Methods in Biomedical Engineering 28(4), 434-455 (2012) 
[8] Blanco, P., Watanabe, S., Feijóo, R.: Identification of vascular territory resistances in one-dimensional hemodynamics simulations. Journal of Biomechanics 45(12), 2066 - 2073 (2012). DOI http://dx.doi.org/10. 1016/j.jbiomech.2012.06.002. URL http://www.sciencedirect.com/ science/article/pii/S002192901200334X

[9] Blanco, P.J., Deparis, S., Malossi, A.C.I.: On the continuity of mean total normal stress in geometrical multiscale cardiovascular problems. Journal of Computational Physics 251(0), 136-155 (2013). DOI http://dx.doi. org/10.1016/j.jcp.2013.05.037. URL http://www.sciencedirect.com/ science/article/pii/S0021999113004038

[10] Blanco, P.J., Feijoo, R.A.: A dimensionally-heterogeneous closed-loop model for the cardiovascular system and its applications. Medical Engineering and Physics 35(5), 652-667 (2013). DOI 10.1016/j.medengphy.2012.07. 011. URL <GotoISI> : //WOS : 000318457200012

[11] Bruneau, C.H., Fabrie, P.: Effective downstream boundary conditions for incompressible Navier-Stokes equations. International Journal for Numerical Methods in Fluids 19(8), 693-705 (1994)

[12] Bruneau, C.H., Fabrie, P.: New efficient boundary conditions for incompressible Navier-Stokes equations: a well-posedness result. RAIRO-Modélisation mathématique et analyse numérique 30(7), 815-840 (1996)

[13] Corsini, C., Baker, C., Kung, E., Schievano, S., Arbia, G., Baretta, A., Biglino, G., Migliavacca, F., Dubini, G., Pennati, G., Marsden, A., VignonClementel, I., Taylor, A., Hsia, T.Y., Dorfman, A., for MOCHA: An integrated approach to patient-specific predictive modeling for single ventricle 
heart palliation. Computer Methods in Biomechanics and Biomedical Engineering pp. 1-18 (2013). DOI 10.1080/10255842.2012.758254. URL http: //www.tandfonline.com/doi/abs/10.1080/10255842.2012.758254

[14] Dobroserdova, T., Olshanskii, M.: A finite element solver and energy stable coupling for 3D and 1D fluid models. Computer Methods in Applied Mechanics and Engineering 259, 166-176 (2013). DOI 10.1016/j.cma.2013.03. 018

[15] Formaggia, L., Gerbeau, J.F., Nobile, F., Quarteroni, A.: On the coupling of 3D and 1D Navier-Stokes equations for flow problems in compliant vessels. Computer Methods in Applied Mechanics and Engineering 191(6-7), 561-582 (2001). DOI http://dx.doi.org/10.1016/S0045-7825(01) 00302-4. URL http://www.sciencedirect.com/science/article/ pii/S0045782501003024

[16] Formaggia, L., Moura, A., Nobile, F.: On the stability of the coupling of 3D and 1D fluid-structure interaction models for blood flow simulations. ESAIM: Mathematical Modelling and Numerical Analysis 41, 743-769 (2007). DOI 10.1051/m2an:2007039

[17] Formaggia, L., Quarteroni, A., Vergara, C.: On the physical consistency between three-dimensional and one-dimensional models in haemodynamics. Journal of Computational Physics 244, 97-112 (2013). DOI 10.1016/j.jcp. 2012.08.001. URL <GotoISI> : //WOS : 000319456900007

[18] Fouchet-Incaux, J.: Artificial boundaries and formulations for the incompressible Navier-Stokes equations: applications to air and blood flows. SeMA Journal 64(1), 1-40 (2014) 
[19] Frank, O.: Die Grundform Des Arteriellen Pulses. Zeitung für Biologie 37, $483-586(1899)$

[20] Gravemeier, V., Comerford, A., Yoshihara, L., Ismail, M., Wall, W.A.: A novel formulation for neumann inflow boundary conditions in biomechanics. International Journal for Numerical Methods in Biomedical Engineering 28(5), 560-573 (2012). DOI 10.1002/cnm.1490. URL <GotoISI> : //WOS : 000303441300005

[21] Guibert, R., Mcleod, K., Caiazzo, A., Mansi, T., Fernández, M.Á., Sermesant, M., Pennec, X., Vignon-Clementel, I., Boudjemline, Y., Gerbeau, J.F.: Group-wise Construction of Reduced Models for Understanding and Characterization of Pulmonary Blood Flows from Medical Images. Medical Image Analysis 18(1), 63-82 (2013). DOI 10.1016/j.media.2013.09.003. URL http://hal.inria.fr/hal-00874545

[22] Heywood, J.G., Rannacher, R., Turek, S.: Artificial boundaries and flux and pressure conditions for the incompressible Navier-Stokes equations. International Journal for Numerical Methods in Fluids 22(5), 325-352 (1996). DOI 10.1002/(sici)1097-0363(19960315)22:5〈325::aid-fld307〉3.0.co;2-y. URL <GotoISI> ://WOS : A1996TY25800001

[23] Ismail, M., Gravemeier, V., Comerford, A., Wall, W.A.: A stable approach for coupling multidimensional cardiovascular and pulmonary networks based on a novel pressure-flow rate or pressure-only neumann boundary condition formulation. International Journal For Numerical Methods in Biomedical Engineering 30(4), 447-469 (2014). DOI 10.1002/cnm.2611

[24] Ismail, M., Wall, W.A., Gee, M.W.: Adjoint-based inverse analysis of windkessel parameters for patient-specific vascular models. Journal of Computa- 
tional Physics 244, 113-130 (2013). DOI 10.1016/j.jcp.2012.10.028. URL <GotoISI> ://WOS :000319456900008

[25] Kim, H.J., Vignon-Clementel, I.E., Figueroa, C.A., Jansen, K.E., Taylor, C.A.: Developing computational methods for three-dimensional finite element simulations of coronary blood flow. Finite Elements in Analysis and Design 46(6), 514-525 (2010). DOI 10.1016/j.finel.2010.01.007. URL <GotoISI> ://WOS:000276019800007

[26] Kuprat, A.P., Kabilan, S., Carson, J.P., Corley, R.A., Einstein, D.R.: A bidirectional coupling procedure applied to multiscale respiratory modeling. Multi-scale Modeling and Simulation of Biological Systems 244(0), 148-167 (2013). URL http://www.sciencedirect.com/scienInce/article/ pii/S0021999112006225

[27] Maury, B., Meunie, N., Soualah, A., Vial, L.: Outlet dissipative conditions for air flow in the bronchial tree. ESAIM: Proc. 14, 201-212 (2005). DOI 10. 1051/proc:2005015. URL http://dx.doi.org/10.1051/proc: 2005015

[28] Migliavacca, F., Balossino, R., Pennati, G., Dubini, G., Hsia, T.Y., de Leval, M.R., Bove, E.L.: Multiscale modelling in biofluidynamics: Application to reconstructive paediatric cardiac surgery. Journal of Biomechanics 39(6), 1010-1020 (2006). DOI 10.1016/j.jbiomech.2005.02.021. URL <GotoISI> : //WOS : 000236834900004

[29] Moghadam, M.E., Bazilevs, Y., Hsia, T.Y., Vignon-Clementel, I.E., Marsden, A.L., for MOCHA: A comparison of outlet boundary treatments for prevention of backflow divergence with relevance to blood flow simulations. Computational Mechanics 48(3), 277-291 (2011). DOI 10.1007/ s00466-011-0599-0. URL <GotoISI> : //WOS : 000294346200004 
[30] Moghadam, M.E., Migliavacca, F., Vignon-Clementel, I.E., Hsia, T.Y., Marsden, A.L., for MOCHA: Optimization of shunt placement for the Norwood surgery using multi-domain modeling. Journal of Biomechanical Engineering-Transactions of the Asme 134(5) (2012). DOI 10.1115/1. 4006814. URL <GotoISI> : //WOS: 000305793100002

[31] Moghadam, M.E., Vignon-Clementel, I.E., Figliola, R., Marsden, A.L., for MOCHA: A modular numerical method for implicit 0D/3D coupling in cardiovascular finite element simulations. Journal of Computational Physics 244, 63-79 (2013). DOI 10.1016/j.jcp.2012.07.035. URL <GotoISI> : //WOS : 000319456900005

[32] Oakes, J., Marsden, A., Grandmont, C., Shadden, S., Darquenne, C., VignonClementel, I.: Airflow and Particle Deposition Simulations in Health and Emphysema: From In Vivo to In Silico Animal Experiments. Annals of Biomedical Engineering 42(4), 899-914 (2014). DOI 10.1007/s10439-013-0954-8. URL http://hal.inria.fr/hal-00916348

[33] Oshima, M., Torii, R., Tokuda, S., Yamada, S., Koizumi, A.: Patient-specific modeling and multi-scale blood simulation for computational hemodynamic study on the human cerebrovascular system. Current Pharmaceutical Biotechnology 13(11), 2153-2165 (2012)

[34] Pant, S., Fabrèges, B., Gerbeau, J.F., Vignon-Clementel, I.: A methodological paradigm for patient-specific multi-scale CFD simulations: from clinical measurements to parameter estimates for individual analysis. International journal for numerical methods in biomedical engineering 30(12), 1614-1648 (2014)

[35] Pant, S., Fabrèges, B., Gerbeau, J.F., Vignon-Clementel, I.: A multiscale 
filtering-based parameter estimation method for patient-specific coarctation simulations in rest and exercise. In: O. Camara, T. Mansi, M. Pop, K. Rhode, M. Sermesant, A. Young (eds.) Statistical Atlases and Computational Models of the Heart. Imaging and Modelling Challenges, Lecture Notes in Computer Science, vol. 8330, pp. 102-109. Springer Berlin Heidelberg (2014). DOI 10.1007/978-3-642-54268-8_12. URL http://dx.doi. org/10.1007/978-3-642-54268-8_12

[36] Porpora, A., Zunino, P., Vergara, C., Piccinelli, M.: Numerical treatment of boundary conditions to replace lateral branches in hemodynamics. International Journal for Numerical Methods in Biomedical Engineering 28(12), 1165-1183 (2012). DOI 10.1002/cnm.2488. URL <GotoISI>://WOS : 000311975400001

[37] Prasad, A., To, L.K., Gorrepati, M.L., Zarins, C.K., Figueroa, C.A.: Computational analysis of stresses acting on intermodular junctions in thoracic aortic endografts. Journal of Endovascular Therapy 18(4), 559-568 (2011). URL <GotoISI > ://WOS :000294212500020

[38] Spilker, R., Taylor, C.: Tuning multidomain hemodynamic simulations to match physiological measurements. Annals of Biomedical Engineering 38(8), 2635-2648 (2010). DOI 10.1007/s10439-010-0011-9. URL http: //dx.doi.org/10.1007/s10439-010-0011-9

[39] Troianowski, G., Taylor, C.A., Feinstein, J.A., Vignon-Clementel, I.E.: Three-dimensional simulations in glenn patients: clinically based boundary conditions, hemodynamic results and sensitivity to input data. Journal of Biomechanical Engineering 133, 111,006 (2011) 
[40] Vignon-Clementel, I.: A coupled multidomain method for computational modeling of blood flow. Ph.D. thesis, Stanford (2006)

[41] Vignon-Clementel, I.E., Figueroa, C.A., Jansen, K.E., Taylor, C.A.: Outflow boundary conditions for three-dimensional finite element modeling of blood flow and pressure in arteries. Computer Methods in Applied Mechanics and Engineering 195(29-32), 3776-3796 (2006). DOI 10.1016/j.cma.2005.04. 014. URL <GotoISI> : //WOS : 000237754700012

[42] Vignon-Clementel, I.E., Figueroa, C.A., Jansen, K.E., Taylor, C.A.: Outflow boundary conditions for 3D simulations of non-periodic blood flow and pressure fields in deformable arteries. Computer Methods in Biomechanics and Biomedical Engineering 13(5), 625-640 (2010). DOI 10.1080/ 10255840903413565. URL <GotoISI> : //WOS : 000281851500012

[43] Vignon-Clementel, I.E., Marsden, A.L., Feinstein, J.A.: A primer on computational simulation in congenital heart disease for the clinician. Progress in Pediatric Cardiology 30(1), 3-13 (2010)

[44] Yang, W., Vignon-Clementel, I.E., Troianowski, G., Reddy, V.M., Feinstein, J.A., Marsden, A.L.: Hepatic blood flow distribution and performance in conventional and novel Y-graft Fontan geometries: A case series computational fluid dynamics study. Journal of Thoracic and Cardiovascular Surgery 143(5), 1086-1097 (2012). DOI 10.1016/j.jtcvs.2011.06.042. URL <GotoISI> : //WOS:000302810700015 